\title{
BERGMAN AND CALDERÓN PROJECTORS FOR DIRAC OPERATORS
}

\author{
COLIN GUILLARMOU, SERGIU MOROIANU, AND JINSUNG PARK
}

\begin{abstract}
For a Dirac operator $D_{\bar{g}}$ over a spin compact Riemannian manifold with boundary $(\bar{X}, \bar{g})$, we give a natural construction of the Calderón projector and of the associated Bergman projector on the space of harmonic spinors on $\bar{X}$, and we analyze their Schwartz kernels. Our approach is based on the conformal covariance of $D_{\bar{g}}$ and the analysis of the complete conformal metric $g=\bar{g} / \rho^{2}$ where $\rho$ is a smooth function on $\bar{X}$ which is equal to the distance to the boundary near $\partial \bar{X}$. We then show that $\frac{1}{2}(\operatorname{Id}+\widetilde{S}(0))$ is the orthogonal Calderón projector, where $\widetilde{S}(\lambda)$ is the holomorphic family in $\{\Re(\lambda) \geq 0\}$ of normalized scattering operators constructed in [24], which are classical pseudo-differential of order $2 \lambda$. Finally we construct natural conformally covariant odd powers of the Dirac operator on any compact spin manifold.
\end{abstract}

\section{INTRODUCTION}

Let $(\bar{X}, \bar{g})$ be a compact spin Riemannian manifold with boundary, and denote by $(M, h)$ its boundary with the induced spin structure and Riemannian metric. Let also $D_{\bar{g}}$ denote the associated Dirac operator acting on the spinor bundle $\Sigma$ over $\bar{X}$. The purpose of this paper is to clarify some aspects of the interaction between the space of smooth spinors of $D_{\bar{g}}$ on $\bar{X}$ which are harmonic in the interior, and the space of their restrictions to the boundary. More precisely, we will examine the orthogonal projectors on these spaces in $L^{2}$ sense, the operator of extension from the boundary to a harmonic spinor, and its adjoint. Before stating our results in the general case, let us review the situation for the unit disc where one can give explicit constructions for these objects.

Example: the unit disc. Keeping the notation $(\bar{X}, \bar{g})$ for the closed unit disc in $\mathbb{C}$ equipped with the Euclidean metric and $M=S^{1}$, let

$$
\mathcal{H}:=\left\{\phi \in C^{\infty}(\bar{X}) ; D_{\bar{g}} \phi=0\right\}, \quad \mathcal{H}_{\partial}:=\left\{\left.\phi\right|_{M} ; \phi \in \mathcal{H}\right\}
$$

where for the moment $D_{\bar{g}}=\bar{\partial}$ is the Cauchy-Riemann operator. The functions $z^{k}, k \geq 0$ clearly are dense in $\mathcal{H}$ with respect to the $L^{2}$ norm. Their restrictions to the boundary $e^{i k t}$, $k \geq 0$, span the space of those smooth functions whose Fourier coefficients corresponding to negative frequencies vanish. The orthogonal projection $P_{\mathcal{H}_{\partial}}$ onto the $L^{2}$-closure of $\mathcal{H}_{\partial}$ is easily seen to be pseudodifferential; if $A=-i d / d t$ is the self-adjoint Dirac operator on $M$, then $P_{\mathcal{H}_{\partial}}$ is the Atiyah-Patodi-Singer projection on the non-negative part of the spectrum of $A$, whose kernel is given by $(2 \pi(1-z \bar{w}))^{-1}$ with respect to the measure $d t$ where $w=e^{i t}$. Let $K: C^{\infty}(M) \rightarrow C^{\infty}(\bar{X})$ be the operator which to $\phi_{\mid M} \in \mathcal{H}_{\partial}$ associates $\phi$, extended by 0 on the orthogonal complement of $\mathcal{H}_{\partial}$. Then $K$ has a smooth kernel on $X \times M$ where $X=\operatorname{int}(\bar{X})$

Date: September 16, 2010. 
given by

$$
K(z, w)=\frac{1}{2 \pi(1-z \bar{w})}
$$

with respect to the standard measure on the circle, where $w=e^{i t}$. This kernel extends to $\bar{X} \times M$ with a singularity at the boundary diagonal $\{(z, w) ; z=w\}$. If we set

$$
z=(1-x) e^{i(t+y)}, \quad \rho:=\sqrt{x^{2}+y^{2}}
$$

we see that the leading term of the singularity is $\rho^{-1}$, moreover $K(z, w)$ admits a power series expansion near $\rho=0$. The coefficients live on the "polar coordinates", or blow-up space which will play an essential role in the rest of this paper. The adjoint of $K$, denoted by $K^{*}$, has a smooth kernel on $M \times X$ with respect to the standard measure $\frac{1}{2 i} d z \wedge d \bar{z}$, given formally by (1). This has the same type of singularity as $K$ near $\{z=w\}$. The kernel of $K^{*} K$ on $M \times M$ is given by

$$
-\frac{1}{4 \pi} \frac{\log (1-z \bar{w})}{z \bar{w}}
$$

which is the kernel of a classical pseudo-differential operator of order -1 (actually given by $\left.\frac{1}{2} P_{\mathcal{H}_{\partial}}\left(\left|D_{t}\right|+1\right)^{-1}\right)$. The remaining composition $K K^{*}$ has a smooth kernel on $X \times X$ given by $(2 \pi(1-z \bar{w}))^{-1}$ with respect to the Euclidean measure in $w$. This kernel extends to $\bar{X} \times \bar{X}$ with the same type of singularity as in the case of $K$ and $K^{*}$, only that now the singular locus is of codimension 3, and there are two, instead of one, extra boundary hyperfaces. To finish our example, consider the projector on (the closure of) $\mathcal{H}$. Its kernel with respect to $\frac{1}{2 i} d w \wedge d \bar{w}$ is

$$
\frac{1}{\pi(1-z \bar{w})^{2}}
$$

which is of the same nature as the kernel of $K K^{*}$ but with a higher order singularity.

Harmonic spinors on manifolds with boundary. One can extend the above example to higher complex dimensions. One direction would be to study holomorphic functions smooth up to the boundary, however in this paper we will consider another generalization. Let thus $\bar{X}$ be a compact domain in $\mathbb{C}^{n}$, and $D_{\bar{g}}=\bar{\partial}+\bar{\partial}^{*}$ acting on $\Lambda^{0, *} X$. A form is called harmonic if it belongs to the nullspace of $D_{\bar{g}}$. Then the above analysis of the operators $K, K^{*}$ and of the projection on the space of harmonic forms can be carried out, describing the singularities of the kernels involved. In fact, even more generally, we will consider the Dirac operator $D_{\bar{g}}$ acting on the spinor bundle $\Sigma$ over a compact spin manifold $\bar{X}$ with boundary $M$. We assume that the metric $\bar{g}$ on $\bar{X}$ is smooth at the boundary but not necessarily of product type (which would mean that the gradient of the distance function $\rho$ to the boundary were Killing near the boundary). We then denote by $\mathcal{H}\left(D_{\bar{g}}\right)$ and $\mathcal{H}_{\partial}\left(D_{\bar{g}}\right)$ the space of smooth harmonic spinors and the Cauchy data space of $D_{\bar{g}}$ respectively,

$$
\mathcal{H}\left(D_{\bar{g}}\right):=\left\{\phi \in C^{\infty}(\bar{X} ; \Sigma) ; D_{\bar{g}} \phi=0\right\}, \quad \mathcal{H}_{\partial}\left(D_{\bar{g}}\right):=\left\{\left.\phi\right|_{M} ; \phi \in \mathcal{H}\left(D_{\bar{g}}\right)\right\}
$$

and let $\overline{\mathcal{H}}\left(D_{\bar{g}}\right)$ and $\overline{\mathcal{H}}_{\partial}\left(D_{\bar{g}}\right)$ be their respective $L^{2}$ closures. When the dependence on $D_{\bar{g}}$ is clear, we may omit $D_{\bar{g}}$ in the notations $\mathcal{H}\left(D_{\bar{g}}\right), \mathcal{H}_{\partial}\left(D_{\bar{g}}\right)$ for simplicity. We denote by $P_{\overline{\mathcal{H}}}$ and $P_{\overline{\mathcal{H}}_{\partial}}$ the respective orthogonal projectors on $\overline{\mathcal{H}}$ (that we call the Bergman projector) and $\overline{\mathcal{H}}_{\partial}$ (the Calderón projector) for the $L^{2}$ inner product induced by $\bar{g}$ and $\left.\bar{g}\right|_{M}$. Let $K$ : $L^{2}(M, \Sigma) \rightarrow L^{2}(\bar{X}, \Sigma)$ be the Poisson operator, i.e. the extension map which sends $\overline{\mathcal{H}}_{\partial}$ to $\overline{\mathcal{H}}$, that is, $D_{\bar{g}} K \psi=0$ and $\left.K \psi\right|_{\partial \bar{X}}=\psi$ for all $\psi \in \mathcal{H}_{\partial}$, and denote by $K^{*}: L^{2}(\bar{X}, \Sigma) \rightarrow L^{2}(M, \Sigma)$ 
its adjoint. The main results in this paper concern the structure of the Schwartz kernels of these operators, which also gives new proofs for some known results.

Let us remark that the construction of the orthogonal projector $P_{\overline{\mathcal{H}}_{\partial}}$ called here Calderón projector, and its applications, have been a central subject in the global analysis of manifolds with boundary since the works of Calderón [9] and Seeley [43], [44]. The Calderón projector of Dirac-type operators turned out to play a fundamental role in geometric problems related to analytic-spectral invariants. This was first observed by Bojarski in the linear conjugation problem of the index of a Dirac type operator [7]. Following Bojarski, Booss and Wojciechowski extensively studied the geometric aspect of the Calderón projector [6]. The Calderón projector also appears in the gluing formulae of the analytic-spectral invariants studied in [40], [42], [28], [32] since the use of the Calderón projector provides us with more refined proofs of these formulae in more general settings. We also refer to [12], [13] for an application of the Calderón projector of the $\operatorname{Spin}_{\mathbb{C}}$ Dirac operator, and a recent paper of Booss-Lesch-Zhu [5] for other generalizations of the work in [6]. Extensions of the Calderón projector for non-smooth boundaries were studied recently in [31].

Polyhomogeneity. Before we state the main results of this paper, let us fix a couple of notations and definitions. If $W$ and $Y$ are smooth compact manifolds (with or without boundary) such that the corner of highest codimension of $W \times Y$ is diffeomorphic to a product $M \times M$ where $M$ is a closed manifold, we will denote, following Mazzeo-Melrose [34], by $W \times{ }_{0} Y$ the smooth compact manifold with corners obtained by blowing-up the diagonal $\Delta$ of $M \times M$ in $W \times Y$, i.e. the manifold obtained by replacing the submanifold $\Delta$ by its interior pointing normal bundle in $W \times Y$ and endowed with the smallest smooth structure containing the lift of smooth functions on $W \times Y$ and polar coordinates around $\Delta$. The bundle replacing the diagonal creates a new boundary hypersurface which we call the front face and we denote by ff. A smooth boundary defining function of ff in $W \times_{0} Y$ can be locally taken to be the lift of $d(\cdot, \Delta)$, the Riemannian distance to the submanifold $\Delta$. On a smooth compact manifold with corners $W$, we say that a function (or distribution) has an integral polyhomogeneous expansion at the boundary hypersurface $H$ if it has an asymptotic expansion at $H$ of the form

$$
\sum_{j=-J}^{\infty} \sum_{\ell=0}^{\alpha(j)} q_{j, \ell} \rho_{H}^{j}\left(\log \rho_{H}\right)^{\ell}
$$

for some $J \in \mathbb{N}_{0}:=\{0\} \cup \mathbb{N}$, a non-decreasing function $\alpha: \mathbb{Z} \rightarrow \mathbb{N}_{0}$, and some smooth functions $q_{j, \ell}$ on $\operatorname{int}(H)$, where $\rho_{H}$ denotes any smooth boundary defining function of $H$ in $W$.

Theorem 1. Let $(\bar{X}, \bar{g})$ be a smooth compact spin Riemannian manifold with boundary $M$. Let $K$ be the Poisson operator for $D_{\bar{g}}$ and let $K^{*}$ be its adjoint. Then the following hold true:

(1) The Schwartz kernels of $K, K^{*}$ and $K K^{*}$ are smooth on the blown-up spaces $\bar{X} \times_{0} M$, $M \times_{0} \bar{X}$, respectively $\bar{X} \times_{0} \bar{X}$ with respect to the volume densities induced by $\bar{g}$.

(2) The operator $K^{*} K$ is a classical pseudo-differential operator of order -1 on $M$ which maps $L^{2}(M, \Sigma)$ to $H^{1}(M, \Sigma)$, and there exists a pseudo-differential operator of order 1 on $M$ denoted by $\left(K^{*} K\right)^{-1}$ such that the Calderón projector $P_{\overline{\mathcal{H}}_{\partial}}$ is given by $\left(K^{*} K\right)^{-1} K^{*} K$. In particular, $P_{\overline{\mathcal{H}}_{\partial}}$ is classical pseudo-differential of order 0 . 
(3) The Bergman orthogonal projection $P_{\overline{\mathcal{H}}}$ from $L^{2}(\bar{X}, \Sigma)$ to $\overline{\mathcal{H}}$ is given by $K\left(K^{*} K\right)^{-1} K^{*}$ and its Schwartz kernel on $\bar{X} \times_{0} \bar{X}$ is smooth except at the front face ff where it has integral polyhomogeneous expansion as in (2) with $\alpha \leq 3$.

Note that an alternate description of these kernels in terms of oscillatory integrals is given in Appendix B.

Our method of proof is to go through an explicit construction of all these operators which does not seem to be written down in the literature in this generality for the Dirac operator, although certainly some particular aspects are well known (especially those involving the Calderón projector $P_{\overline{\mathcal{H}}_{\partial}}$, see [6]). We use the fundamental property that the Dirac operator is conformally covariant to transform the problem into a problem on a complete non-compact manifold $(X, g)$ conformal to $(\bar{X}, \bar{g})$ obtained by simply considering $X:=\operatorname{int}(\bar{X})$ and $g:=\bar{g} / \rho^{2}$ where $\rho$ is a smooth boundary defining function of the boundary $M=\partial \bar{X}$ which is equal to the distance to the boundary (for the metric $\bar{g}$ ) near $\partial \bar{X}$. This kind of idea is not really new since this is also in spirit used for instance to study pseudoconvex domains by considering a complete Kähler metric in the interior of the domain (see Donnelly-Fefferman [11], Fefferman [15], Cheng-Yau [10], Epstein-Melrose [14]), and obviously this connection is transparent for the disc in $\mathbb{C}$ via the Poisson kernel and the relations with the hyperbolic plane. One of the merits of this method, for instance, is that we do not need to go through the invertible double of [6] to construct the Calderón projector and thus we do not need the product structure of the metric near the boundary. We finally remark that the bound $\alpha \leq 3$ in (3) of the Theorem is almost certainly not optimal, we expect instead $\alpha \leq 1$ to be true.

Conformally covariant operators. We also obtain, building on our previous work [24],

Theorem 2. There exists a holomorphic family in $\{\lambda \in \mathbb{C} ; \Re(\lambda) \geq 0\}$ of elliptic pseudodifferential operators $\widetilde{S}(\lambda)$ on $M=\partial \bar{X}$ of complex order $2 \lambda$, invertible except at a discrete set of $\lambda$ 's and with principal symbol $i \operatorname{cl}(\nu) \operatorname{cl}(\xi)|\xi|^{2 \lambda-1}$ where $\nu$ is the inner unit normal vector field to $M$ with respect to $\bar{g}$, such that

(a) $\frac{1}{2}(\mathrm{Id}+\widetilde{S}(0))$ is the Calderón projector $P_{\overline{\mathcal{H}}_{\partial}}$;

(b) For $k \in \mathbb{N}_{0}, L_{k}:=-\operatorname{cl}(\nu) \widetilde{S}(1 / 2+k)$ is a conformally covariant differential operator whose leading term is $D_{M}^{1+2 k}$ where $D_{M}$ denotes the Dirac operator on $M$, and $L_{0}=$ $D_{M}$.

By using the existence of ambient (or Poincaré-Einstein) metric of Fefferman-Graham [16, 17], this leads to the construction of natural conformally covariant powers of Dirac operators in degree $2 k+1$ on any spin Riemannian manifolds $(M, h)$ of dimension $n$, for all $k \in \mathbb{N}_{0}$ if $n$ is odd and for $k \leq n / 2$ if $n$ is even. We explicitly compute $L_{1}$.

Corollary 3. Let $(M, h)$ be a Riemannian manifold of dimension $n \geq 3$ with a fixed spin structure, and denote by scal, Ric and $D$ the scalar curvature, the Ricci curvature, and respectively the Dirac operator with respect to $h$. Then the operator $L_{1}$ defined by

$$
L_{1}:=D^{3}-\frac{\operatorname{cl}(d(\text { scal }))}{2(n-1)}-\frac{2 \operatorname{cl} \circ \operatorname{Ric} \circ \nabla}{n-2}+\frac{\text { scal }}{(n-1)(n-2)} D
$$

is a natural conformally covariant differential operator:

$$
\hat{L}_{1}=e^{-\frac{n+3}{2} \omega} L_{1} e^{\frac{n-3}{2}}
$$

if $\hat{L}_{1}$ is defined in terms of the conformal metric $\hat{h}=e^{2 \omega} h$. 
Cobordism invariance of the index and local Wodzicki-Guillemin residue for the Calderón projector. As a consequence of Theorem 2 and the analysis of [24], we deduce the following

Corollary 4. Let $(\bar{X}, \bar{g})$ be a smooth compact spin Riemannian manifold with boundary $M$.

(1) The Schwartz kernel of the Calderón projector $P_{\overline{\mathcal{H}}_{a}}$ associated to the Dirac operator has an asymptotic expansion in polar coordinates around the diagonal without log terms. In particular, the Wodzicki-Guillemin local residue density of $P_{\overline{\mathcal{H}}_{\partial}}$ vanishes.

(2) When the dimension of $M$ is even, the spinor bundle $\Sigma$ splits in a direct sum $\Sigma_{+} \oplus \Sigma_{-}$. If $D_{M}^{+}$denotes $\left.D_{M}\right|_{\Sigma_{+}}: \Sigma_{+} \rightarrow \Sigma_{-}$, then the index $\operatorname{Ind}\left(D_{M}^{+}\right)$is 0 .

As far as we know, the first part of the corollary is new. It is known since Wodzicki [45] that the global residue trace of a pseudo-differential projector of order 0 vanishes, however the local residue density does not vanish for general projectors (e.g. see [18]). What is true is that the APS spectral projector has also vanishing local residue, a fact which is equivalent to the conformal invariance of the eta invariant. For metrics of product type near the boundary the Calderón and APS projectors coincide up to smoothing operators; thus our result was known for such metrics.

The second statement is the well-known cobordism invariance of the index for the Dirac operator; there exist several proofs of this fact for more general Dirac type operators (see for instance $[1,38,30,41,8])$ but we found it worthwhile to point out that this fact can be obtained as a easy consequence of the invertibility of the scattering operator. In fact, a proof of cobordism invariance using scattering theory for cylindrical metrics has been found recently by Müller-Strohmaier [39], however their approach does not seem to have implications about the Caldéron or Bergman projectors.

More general operators. Our approach does not seem to work for more general Dirac type operators. However it applies essentially without modifications to twisted spin Dirac operators, with twisting bundle and connection smooth on $\bar{X}$. For simplicity of notation, we restrict ourselves to the untwisted case.

Acknowledgements. This project was started while the first two authors were visiting KIAS Seoul, it was continued while C.G. was visiting IAS Princeton, and finished while S.M. was visiting ENS Paris; we thank these institutions for their support. We also thank Andrei Moroianu for checking (with an independent method) the formula for $L_{1}$ in Corollary 3. C.G. was supported by the grant NSF-0635607 at IAS. S.M. was supported by the grant PN-II-ID-PCE 1188 265/2009 and by a CNRS grant at ENS.

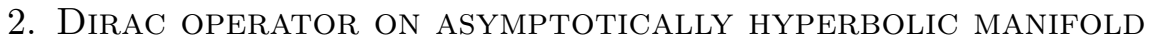

We start by recalling the results of [24] that we need for our purpose. Let $(X, g)$ be an $(n+1)$-dimensional smooth complete non-compact spin manifold which is the interior of a smooth compact manifold with boundary $\bar{X}$. We shall say that it is asymptotically hyperbolic if the metric $g$ has the following properties: there exists a smooth boundary defining function $x$ of $\partial \bar{X}$ such that $x^{2} g$ is a smooth metric on $\bar{X}$ and $|d x|_{x^{2} g}=1$ at $\partial \bar{X}$. It is shown in $[19,27]$ that for such metrics, there is a diffeomorphism $\psi:[0, \epsilon)_{t} \times \partial \bar{X} \rightarrow U \subset \bar{X}$ such that

$$
\psi^{*} g=\frac{d t^{2}+h(t)}{t^{2}}
$$


where $\epsilon>0$ is small, $U$ is an open neighborhood of $\partial \bar{X}$ in $\bar{X}$ and $h(t)$ is a smooth oneparameter family of metrics on $\partial \bar{X}$. The function $\psi_{*}(t)$ will be called geodesic boundary defining function of $\partial \bar{X}$ and the metric $g$ will be said even to order $2 k+1$ if $\partial_{t}^{2 j+1} h(0)=0$ for all $j<k$; such a property does not depend on $\psi$, as it is shown in [22]. The conformal infinity of $\bar{X}$ is the conformal class on $\partial \bar{X}$ given by

$$
\left[h_{0}\right]:=\left\{\left.\left(x^{2} g\right)\right|_{T \partial \bar{X}} ; x \text { is a boundary defining function of } \bar{X}\right\} .
$$

On $\bar{X}$ there exists a natural smooth bundle ${ }^{0} T \bar{X}$ whose space of smooth sections is canonically identified with the Lie algebra $\mathcal{V}_{0}$ of smooth vector fields which vanish at the boundary $\partial \bar{X}$, its dual ${ }^{0} T^{*} \bar{X}$ is also a smooth bundle over $\bar{X}$ and $g$ is a smooth metric on ${ }^{0} T \bar{X}$.

Consider the $\mathrm{SO}(n+1)$-principal bundle ${ }_{o}^{0} F(\bar{X}) \rightarrow \bar{X}$ over $\bar{X}$ of orthonormal frames in ${ }^{0} T \bar{X}$ with respect to $g$. Since $\bar{X}$ is spin, there is a $\operatorname{Spin}(n+1)$-principal bundle ${ }_{s}^{0} F(\bar{X}) \rightarrow \bar{X}$ which double covers ${ }_{o}^{0} F(\bar{X})$ and is compatible with it in the usual sense. The 0-Spinor bundle ${ }^{0} \Sigma(\bar{X})$ can then be defined as a bundle associated to the $\operatorname{Spin}(n+1)$-principal bundle ${ }_{s}^{0} F(\bar{X})$, with the fiber at $p \in \bar{X}$

$$
{ }^{0} \Sigma_{p}(\bar{X})=\left({ }_{s}^{0} F_{p} \times S(n+1)\right) / \tau
$$

where $\tau: \operatorname{Spin}(n+1) \rightarrow \operatorname{Hom}(S(n+1))$ is the standard spin representation on $S(n+1) \simeq$ $\mathbb{C}^{2^{[(n+1) / 2]}}$. If $x$ is any geodesic boundary defining function, the unit vector field $x \partial_{x}:=$ $\nabla^{g} \log (x)$ is a smooth section of ${ }^{0} T \bar{X}$. The Clifford multiplication $\operatorname{cl}\left(x \partial_{x}\right)$ restricts to the boundary to a map denoted by $\operatorname{cl}(\nu)$, independent of the choice of $x$, satisfying $\operatorname{cl}(\nu)^{2}=-\mathrm{Id}$ which splits the space of 0 -spinors on the boundary into $\pm i$ eigenspaces

$$
{ }^{0} \Sigma_{ \pm}:=\operatorname{ker}(\operatorname{cl}(\nu) \mp i),\left.\quad{ }^{0} \Sigma\right|_{M}={ }^{0} \Sigma_{+} \oplus{ }^{0} \Sigma_{-}
$$

The Dirac operator $D_{g}$ associated to $g$ acts in $L^{2}\left(X,{ }^{0} \Sigma\right)$ and is self-adjoint since the metric $g$ is complete. Let us denote by $\dot{C}^{\infty}\left(\bar{X},{ }^{0} \Sigma\right)$ the set of smooth spinors on $\bar{X}$ which vanish to infinite order at $\partial \bar{X}$. We proved the following result in [24, Prop 3.2]:

Proposition 5. The spectrum of $D_{g}$ is absolutely continuous and given by the whole real line $\sigma\left(D_{g}\right)=\mathbb{R}$. Moreover the $L^{2}$ bounded resolvent $R_{ \pm}(\lambda):=\left(D_{g} \pm i \lambda\right)^{-1}$ extends from $\{\Re(\lambda)>0\}$ meromorphically in $\lambda \in \mathbb{C} \backslash-\mathbb{N} / 2$ as a family of operators mapping $\dot{C}^{\infty}\left(\bar{X},{ }^{0} \Sigma\right)$ to $x^{\frac{n}{2}+\lambda} C^{\infty}\left(\bar{X},{ }^{0} \Sigma\right)$, and it is analytic in $\{\Re(\lambda) \geq 0\}$. Finally, we have $\left.\left[x^{-\frac{n}{2}-\lambda} R_{ \pm}(\lambda) \sigma\right]\right|_{\partial \bar{X}} \in$ $C^{\infty}\left(\partial \bar{X},{ }^{0} \Sigma_{\mp}\right)$ for all $\sigma \in \dot{C}^{\infty}\left(\bar{X},{ }^{0} \Sigma\right)$.

Using this result, in [24] we were able to solve the following boundary value problem

Proposition 6. Let $\lambda \in U:=\{z \in \mathbb{C} ; \Re(z) \geq 0, z \notin \mathbb{N} / 2\}$. For all $\psi \in C^{\infty}\left(\partial \bar{X},{ }^{0} \Sigma_{ \pm}\right)$there is a unique $\sigma_{ \pm}(\lambda) \in C^{\infty}\left(X,{ }^{0} \Sigma\right)$ such that there exist $\sigma_{ \pm}^{+}(\lambda), \sigma_{ \pm}^{-}(\lambda) \in C^{\infty}\left(\bar{X},{ }^{0} \Sigma\right)$ satisfying $\sigma_{ \pm}(\lambda)=x^{\frac{n}{2}-\lambda} \sigma_{ \pm}^{-}(\lambda)+x^{\frac{n}{2}+\lambda} \sigma_{ \pm}^{+}(\lambda)$ and

$$
\left(D_{g} \pm i \lambda\right) \sigma_{ \pm}(\lambda)=0,\left.\quad \sigma_{ \pm}^{-}(\lambda)\right|_{\partial \bar{X}}=\psi .
$$

Moreover $\sigma_{ \pm}^{+}(\lambda), \sigma_{ \pm}^{-}(\lambda)$ are analytic in $\lambda \in U$ and one has $\left.\sigma_{ \pm}^{+}(\lambda)\right|_{\partial \bar{X}} \in C^{\infty}\left(\partial \bar{X},{ }^{0} \Sigma_{\mp}\right)$.

The solution $\sigma_{ \pm}(\lambda)$ of Proposition 6 is constructed in Lemma 4.4 of [24] as a sum

$$
\sigma_{ \pm}(\lambda)=\sigma_{\infty, \pm}(\lambda)-R_{ \pm}(\lambda)\left(D_{g} \pm i \lambda\right) \sigma_{\infty, \pm}(\lambda)
$$

where $\sigma_{\infty, \pm}(\lambda) \in x^{\frac{n}{2}-\lambda} C^{\infty}\left(\bar{X},{ }^{0} \Sigma\right)$ satisfies

$$
\left(D_{g} \pm i \lambda\right) \sigma_{\infty, \pm}(\lambda) \in \dot{C}^{\infty}\left(\bar{X},{ }^{0} \Sigma\right)
$$

$$
\left.\left[x^{-\frac{n}{2}+\lambda} \sigma_{\infty, \pm}(\lambda)\right]\right|_{\partial \bar{X}}=\psi
$$


with the additional property that it is analytic in $\{\Re(\lambda) \geq 0, \lambda \notin \mathbb{N} / 2\}$. Since $R_{ \pm}(\lambda)$ are analytic in $\{\Re(\lambda) \geq 0\}$, this shows that $\sigma_{ \pm}(\lambda)$ is analytic in the same domain, and we have $D_{g} \sigma_{ \pm}(0)=0$. Since this will be useful below, we recall briefly the construction of the approximate solution $\sigma_{\infty, \pm}(\lambda)$ near the boundary from Lemma 4.4 in [24]. The principle is to write the Dirac operator near $\partial \bar{X}$ in the product decomposition $[0, \epsilon)_{x} \times \partial \bar{X}$

$$
D_{g}=x^{\frac{n}{2}}\left(\operatorname{cl}\left(x \partial_{x}\right) x \partial_{x}+x D_{h_{0}}\right) x^{-\frac{n}{2}}+x P
$$

where $D_{h_{0}}$ is the Dirac operator on the boundary for the metric $h_{0}$ and $P$ is a first order differential operator with smooth coefficients which in local coordinates $(x, y)$ near the boundary can be written

$$
P=P_{0}(x, y) x \partial_{x}+\sum_{j=1}^{n} P_{j}(x, y) x \partial_{y_{i}}
$$

for some smooth sections $P_{j}$ of ${ }^{0} \Sigma \otimes{ }^{0} \Sigma^{*}$. Consequently, one has for any $\psi_{ \pm} \in C^{\infty}\left(\partial \bar{X},{ }^{0} \Sigma_{ \pm}\right)$ and $k \in \mathbb{N}_{0}$ the indicial equation

$$
\begin{aligned}
& \left(D_{g} \pm i \lambda\right) x^{\frac{n}{2}-\lambda+k}\left(\psi_{+}+\psi_{-}\right) \\
& \quad=i x^{\frac{n}{2}-\lambda+k}\left((k-\lambda \pm \lambda) \psi_{+}+(\lambda-k \pm \lambda) \psi_{-}\right)+x^{\frac{n}{2}-\lambda+k+1} F_{\lambda}^{k}
\end{aligned}
$$

where $F_{\lambda}^{k} \in C^{\infty}\left(\bar{X},{ }^{0} \Sigma\right)$ is holomorphic near $\lambda=0$. From this, using formal series and Borel lemma, it is easy to see that one can construct near $\lambda=0$ a spinor $\sigma_{\infty, \pm}(\lambda) \in x^{\frac{n}{2}-\lambda} C^{\infty}\left(\bar{X},{ }^{0} \Sigma\right)$, holomorphic near $\lambda=0$, solving (6) whose formal Taylor series is determined locally and uniquely by $\psi_{ \pm}$.

Let $\sigma_{ \pm}(\lambda)$ be the spinor of Proposition 6 (thus depending on $\psi$ ), we can then define linear Poisson operators and scattering operators

$$
\begin{array}{rlrl}
E_{ \pm}(\lambda): C^{\infty}\left(\partial \bar{X},{ }^{0} \Sigma_{ \pm}\right) & \rightarrow C^{\infty}\left(X,{ }^{0} \Sigma\right), & & \psi \mapsto \sigma_{ \pm}(\lambda), \\
S_{ \pm}(\lambda): C^{\infty}\left(\partial \bar{X},{ }^{0} \Sigma_{ \pm}\right) \rightarrow C^{\infty}\left(\partial \bar{X},{ }^{0} \Sigma_{\mp}\right), & & \left.\psi \mapsto \sigma_{ \pm}^{+}(\lambda)\right|_{\partial \bar{X}}
\end{array}
$$

which are holomorphic in $\{\Re(\lambda) \geq 0, \lambda \notin \mathbb{N} / 2\}$. We extend the definition of $E_{ \pm}(\lambda)$ to the whole bundle ${ }^{0} \Sigma$ by setting that it acts by 0 on ${ }^{0} \Sigma_{\mp}$. Then from Proposition 4.6 of [24], the Schwartz kernel $E_{ \pm}\left(\lambda ; m, y^{\prime}\right) \in C^{\infty}\left(X \times \partial \bar{X} ;{ }^{0} \Sigma \otimes{ }^{0} \Sigma^{*}\right)$ of $E_{ \pm}(\lambda)$ is given by

$$
E_{ \pm}\left(\lambda ; m, y^{\prime}\right)=\left.\left[R_{ \pm}\left(\lambda ; m, x^{\prime}, y^{\prime}\right) x^{\prime-\frac{n}{2}-\lambda}\right]\right|_{x^{\prime}=0} \operatorname{cl}(\nu)
$$

where $R_{ \pm}\left(\lambda ; m, m^{\prime}\right)$ is the Schwartz kernel of $R_{ \pm}(\lambda)$. We can also define

$$
\begin{array}{rlrl}
E(\lambda): C^{\infty}\left(\partial \bar{X},{ }^{0} \Sigma\right) & \rightarrow C^{\infty}\left(X,{ }^{0} \Sigma\right), & \psi_{+}+\psi_{-} \mapsto E_{+}(\lambda) \psi_{+}+E_{-}(\lambda) \psi_{-}, \\
S(\lambda): C^{\infty}\left(\partial \bar{X},{ }^{0} \Sigma\right) \rightarrow C^{\infty}\left(\partial \bar{X},{ }^{0} \Sigma\right) & \psi_{+}+\psi_{-} \mapsto S_{+}(\lambda) \psi_{+}+S_{-}(\lambda) \psi_{-} .
\end{array}
$$

The main features of $S(\lambda)$, also proved in Section 4.3 of [24], are gathered in

Proposition 7. For $\Re(\lambda) \geq 0$ and $\lambda \notin \mathbb{N} / 2$, the operator $S(\lambda)$ depends on the choice of the boundary defining function $x$ but changes under the law

$$
\hat{S}(\lambda)=e^{-\left(\frac{n}{2}+\lambda\right) \omega_{0}} S(\lambda) e^{\left(\frac{n}{2}-\lambda\right) \omega_{0}}, \quad \omega_{0}:=\left.\omega\right|_{x=0}
$$

if $\hat{S}(\lambda)$ is the scattering operator defined using the boundary defining function $\hat{x}=e^{\omega} x$ for some $\omega \in C^{\infty}(\bar{X})$. Moreover $S(\lambda) \in \Psi^{2 \lambda}\left(\partial \bar{X},{ }^{0} \Sigma\right)$ is a classical pseudodifferential operator of 
order $2 \lambda$, and its principal symbol is given by

$$
\sigma_{\operatorname{pr}}(S(\lambda))(\xi)=i 2^{-2 \lambda} \frac{\Gamma(1 / 2-\lambda)}{\Gamma(1 / 2+\lambda)} \operatorname{cl}(\nu) \operatorname{cl}(\xi)|\xi|_{h_{0}}^{2 \lambda-1}
$$

where $h_{0}=\left.\left(x^{2} g\right)\right|_{T \partial \bar{X}}$. If $\lambda \in i \mathbb{R}, S(\lambda)$ extends as a unitary operator on $L^{2}\left(\partial \bar{X},{ }^{0} \Sigma\right)$, its inverse is given by $S(-\lambda)$ and extends meromorphically in $\{\Re(\lambda) \geq 0, \lambda \notin \mathbb{N} / 2\}$ as a family of classical pseudo-differential operators in $\Psi^{-2 \lambda}\left(\partial \bar{X},{ }^{0} \Sigma\right)$. Finally $S(\lambda)$ is self-adjoint for $\lambda \in(0, \infty)$.

The conformal change law and the invertibility are easy consequences of the definition of $S(\lambda)$ and the uniqueness of the solution $\sigma_{ \pm}(\lambda)$ in Proposition 6 , the pseudodifferential properties and the meromorphic extension are more delicate and studied in Section 4.3 of [24]. In particular, by letting $\lambda \rightarrow 0$ in (4), we deduce easily the following

Proposition 8. Let $\psi \in C^{\infty}\left(\partial \bar{X},{ }^{0} \Sigma\right)$, then $\sigma:=E(0) \psi$ is a harmonic spinor for $D$, which lives in $x^{\frac{n}{2}} C^{\infty}\left(\bar{X},{ }^{0} \Sigma\right)$ and has the following behavior at the boundary

$$
\sigma=x^{\frac{n}{2}}(\mathrm{Id}+S(0)) \psi+O\left(x^{\frac{n}{2}+1}\right) .
$$

Remark from Proposition 7 that $S(0)^{*}=S(0)^{-1}=S(0)$ and so the operator

$$
\mathrm{C}:=\frac{1}{2}(\mathrm{Id}+S(0))
$$

is an orthogonal projector on a subspace of $L^{2}\left(\partial \bar{X},{ }^{0} \Sigma\right)$ for the measure $\mathrm{dv}_{h_{0}}$ where $h_{0}=$ $\left.\left(x^{2} g\right)\right|_{T \partial \bar{X}}$. Notice from (11) that, under a change of boundary defining function $\hat{x}=e^{\omega} x$, the operator $\mathcal{C}$ changes according to conjugation $\hat{\mathcal{C}}=e^{-\frac{n}{2} \omega_{0}} \mathcal{C} e^{\frac{n}{2} \omega_{0}}$.

Now we want to prove that the range of $E(0)$ acting on $C^{\infty}\left(\partial \bar{X},{ }^{0} \Sigma\right)$ is exactly the set of harmonic spinors in $x^{\frac{n}{2}} C^{\infty}\left(\bar{X},{ }^{0} \Sigma\right)$.

Proposition 9. Let $\phi \in x^{\frac{n}{2}} C^{\infty}\left(\bar{X},{ }^{0} \Sigma\right)$ such that $D_{g} \phi=0$ and let $\psi:=\left.\left(x^{-\frac{n}{2}} \phi\right)\right|_{\partial \bar{X}}$. Then we have $E(0) \psi=2 \phi$.

Proof. First let us write $\psi=\psi_{+}+\psi_{-}$with $\psi_{ \pm} \in{ }^{0} \Sigma_{ \pm}$. Then we construct the approximate solution $\sigma_{\infty,+}(\lambda)$ of $(6)$ associated to $\psi_{+}$. Let us set $\phi_{+}(\lambda):=\sigma_{\infty,+}(\lambda)$ and $\phi_{-}(\lambda):=\phi-$ $\phi_{+}(\lambda)$. One has $\left.\left(x^{-\frac{n}{2}} \phi_{-}(0)\right)\right|_{x=0}=\psi_{-} \in{ }^{0} \Sigma_{-}$and $D_{g} \phi_{-}(0)=-D_{g} \phi_{+}(0)$. As in the proof of Proposition 6, we have

$$
\sigma_{+}(\lambda)=\phi_{+}(\lambda)-R_{+}(\lambda)\left(D_{g}+i \lambda\right) \phi_{+}(\lambda)=E_{+}(\lambda) \psi_{+} .
$$

and in particular, since all the terms in the composition on the right hand side are holomorphic near $\lambda=0$, we obtain that

$$
E_{+}(0) \psi_{+}=\phi_{+}(0)-R_{+}(0) D_{g} \phi_{+}(0)=\phi_{+}(0)+R_{+}(0) D_{g} \phi_{-}(0) .
$$

Now we use Green's formula on a region $\{x \leq \epsilon\}$ for $\epsilon>0$ small and by letting $\epsilon \rightarrow 0$ we deduce easily from (9) that

$$
R_{+}(0) D_{g} \phi_{-}(0)=\phi_{-}(0)-E_{+}(0) \psi_{-}=\phi_{-}(0) .
$$

Consequently, we have proved that $E_{+}(0) \psi_{+}=\phi_{+}(0)+\phi_{-}(0)=\phi$. A similar reasoning shows that $E_{-}(0) \psi_{-}=\phi$ and this achieves the proof.

As a corollary we deduce that $S(0) \psi=\psi$ for $\psi$ as in Proposition 9, so 
Theorem 10. The following identity holds for $\mathrm{C}=\frac{1}{2}(\mathrm{Id}+S(0))$

$$
\left\{\left.\left(x^{-\frac{n}{2}} \sigma\right)\right|_{\partial \bar{X}} ; \sigma \in x^{\frac{n}{2}} C^{\infty}\left(\bar{X},{ }^{0} \Sigma\right), D_{g} \sigma=0\right\}=\left\{\mathrm{e} \psi ; \psi \in C^{\infty}\left(\partial \bar{X},{ }^{0} \Sigma\right)\right\} .
$$

\section{DiRAC OPERATOR ON COMPACT MANIFOLDS WITH BOUNDARY}

3.1. Calderón projector and scattering operator at 0 . Now we let $D_{\bar{g}}$ be the Dirac operator on a smooth compact spin manifold with boundary $(\bar{X}, \bar{g})$, and we denote by $\Sigma$ the spinor bundle. We recall that the Cauchy data space of $D_{\bar{g}}$ is given by

$$
\mathcal{H}_{\partial}:=\left\{\left.\phi\right|_{\partial \bar{X}}, \phi \in C^{\infty}(\bar{X}, \Sigma), D_{\bar{g}} \phi=0\right\}
$$

i.e. it is the space of boundary values of smooth harmonic spinors on $\bar{X}$ for $D_{\bar{g}}$. The orthogonal Calderón projector $P_{\overline{\mathcal{H}_{\partial}}}$ is a projector acting on $L^{2}(\partial \bar{X}, \Sigma)$ and whose range is the $L^{2}$-closure $\overline{\mathcal{H}}_{\partial}$. Booss and Wojciechowski [6] studied Fredholm properties of boundary value problems for Dirac type operators on manifolds with boundary, they found that if $P$ is a pseudo-differential projector on the boundary, the operator $D_{P}^{+}: \operatorname{Dom}\left(D_{P}^{+}\right) \rightarrow C^{\infty}\left(\bar{X}, \Sigma^{+}\right)$with domain

$$
\operatorname{Dom}\left(D_{P}^{+}\right):=\left\{\phi \in C^{\infty}\left(\bar{X}, \Sigma^{+}\right) ; P\left(\left.\phi\right|_{\partial \bar{X}}\right)=0\right\}
$$

is Fredholm if and only if $P \circ P_{\overline{\mathcal{H}}_{\partial}}: \mathcal{H}_{\partial} \rightarrow \operatorname{ran}(P)$ is Fredholm, and their indices agree. One of the main problems in this setting is to construct Calderón projectors, there exist methods by Wojciechowski [6] which use the invertible double construction, but a special product structure near the boundary has to be assumed. Our purpose is to construct the Calderón projector in a general setting for the Dirac operator using its conformal covariance and the scattering theory of Dirac operators on asymptotically hyperbolic manifolds developed in [24].

Let $x$ be the distance to the boundary, which is smooth near $\partial \bar{X}$, and modify it on a compact set of $X$ so that it becomes smooth on $\bar{X}$, we still denote it by $x$. Define a metric $g$ conformal to $\bar{g}$ by

$$
g:=x^{-2} \bar{g}
$$

this is a complete metric on the interior $X$ which is asymptotically hyperbolic. The associated Dirac operator $D$ is related to $D_{\bar{g}}$ by the conformal law change

$$
D_{g}=x^{\frac{n}{2}+1} D_{\bar{g}} x^{-\frac{n}{2}} .
$$

Notice that this formula appears with a wrong exponent in several places in the literature, e.g. [25, Prop. 1.3], [29, Thm. II.5.24]. Let ${ }^{0} \Sigma$ be the rescaled spin bundle defined in Section 2 , then there is a canonical identification between $\Sigma$ and ${ }^{0} \Sigma$. We deduce that the Cauchy data space may also be given by

$$
\mathcal{H}_{\partial}=\left\{\left.\left(x^{-\frac{n}{2}} \sigma\right)\right|_{\partial \bar{X}} ; \sigma \in x^{\frac{n}{2}} C^{\infty}\left(\bar{X},{ }^{0} \Sigma\right), D_{g} \sigma=0\right\} .
$$

Combining this and Theorem 10, we obtain

Theorem 11. The $L^{2}$-closure of the Cauchy data space $\overline{\mathcal{H}}_{\partial}$ is given by the range of $\mathrm{C}=$ $\frac{1}{2}(\mathrm{Id}+S(0))$ on $L^{2}\left(\partial \bar{X},{ }^{0} \Sigma\right)$, in particular, $P_{\overline{\mathcal{H}}_{\partial}}=\mathrm{C}$.

Remark that no assumption is needed on the geometry of $(\bar{X}, \bar{g})$ (this was needed for instance for the double construction in [6]).

Another consequence of our construction is that $S(0)$ anti-commutes with the endomor$\operatorname{phism} \operatorname{cl}(\nu)$ of Section 2 and thus 
Proposition 12. The operator $\mathcal{C}$ satisfies $-\operatorname{cl}(\nu) \mathcal{C} \operatorname{cl}(\nu)=\operatorname{Id}-\mathcal{C}$, in other words, the $L^{2}$ closure of the Cauchy data space $\overline{\mathcal{H}}_{\partial}$ is a Lagrangian subspace in $L^{2}\left(\partial \bar{X},{ }^{0} \Sigma\right)$ with respect to the symplectic structure $(v, w):=\langle\operatorname{cl}(\nu) v, w\rangle_{h_{0}}$ for $v, w \in L^{2}\left(\partial \bar{X},{ }^{0} \Sigma\right)$ where $h_{0}=\left.(\bar{g})\right|_{T \partial \bar{X}}$.

Proof. The equality $-\operatorname{cl}(\nu) \mathcal{C} \operatorname{cl}(\nu)=\mathrm{Id}-\mathcal{C}$ follows easily from $\operatorname{cl}(\nu) \mathrm{S}(0)=-\mathrm{S}(0) \operatorname{cl}(\nu)$ since

$$
-\frac{1}{2} \operatorname{cl}(\nu)(\operatorname{Id}+\mathrm{S}(0)) \operatorname{cl}(\nu)=\frac{1}{2}(\operatorname{Id}-\operatorname{cl}(\nu) \mathrm{S}(0) \operatorname{cl}(\nu))=\frac{1}{2}(\mathrm{Id}-\mathrm{S}(0)) .
$$

This immediately implies that $\overline{\mathcal{H}}_{\partial}$ and $\overline{\mathcal{H}}^{\perp}$ are both isotropic subspaces in $L^{2}\left(\partial \bar{X},{ }^{0} \Sigma\right)$, which completes the proof.

3.2. Calderón projector and the operator $K$. By Propositions 8 and 9 , the extension map $K: C^{\infty}\left(\partial \bar{X},{ }^{0} \Sigma\right) \rightarrow C^{\infty}\left(\bar{X},{ }^{0} \Sigma\right)$ from spinors on $M$ to harmonic spinors on $\bar{X}$ is given by

$$
K \psi=\frac{1}{2} x^{-\frac{n}{2}} E(0) \psi
$$

where $E(0)$ is the operator defined in (10) for the Dirac operator $D$ associated to $g=\bar{g} / x^{2}$. The adjoint $E(0)^{*}$ of $E(0)$ with respect to $\mathrm{dv}_{g}$ is a map from $\dot{C}^{\infty}\left(\bar{X},{ }^{0} \Sigma\right)$ to $C^{\infty}\left(\partial \bar{X},{ }^{0} \Sigma\right)$ such that

$$
\int_{X}\langle E(0) \varphi, \psi\rangle_{g} \mathrm{dv}_{g}=\int_{\partial \bar{X}}\left\langle\varphi, E(0)^{*} \psi\right\rangle_{h_{0}} \mathrm{dv}_{h_{0}}
$$

for all $\psi \in \dot{C}^{\infty}\left(\bar{X},{ }^{0} \Sigma\right)$ and $\varphi \in C^{\infty}\left(\partial \bar{X},{ }^{0} \Sigma\right)$. Here $h_{0}$ denotes the metric over $\partial \bar{X}$ given by the restriction of $\bar{g}$ to the bundle $T \partial \bar{X}$. Similarly the adjoint of $K$ with respect to the metric $\mathrm{dv}_{\bar{g}}$ satisfies

and since $\mathrm{dv}_{g}=x^{-(n+1)} \mathrm{dv}_{\bar{g}}$, we obtain

$$
\int_{X}\langle K \varphi, \psi\rangle_{g} \mathrm{dv}_{\bar{g}}=\int_{\partial \bar{X}}\left\langle\varphi, K^{*} \psi\right\rangle_{h_{0}} \mathrm{dv}_{h_{0}}
$$

$$
K^{*}=\frac{1}{2} E(0)^{*} x^{\frac{n}{2}+1}
$$

where the adjoint for $E(0)$ is with respect to $g$ while the adjoint for $K$ is with respect to $\bar{g}$.

The Schwartz kernels of $E(\lambda), E^{*}(\lambda)$ and $R_{ \pm}(\lambda)$ are studied in [24]. They are shown to be polyhomogeneous conormal on a blown-up space. Let us now describe them, by referring the reader to the Appendix for what concerns blown-up manifolds and polyhomogeneous conormal distributions. The first space is the stretched product (see for instance [34, 33] where it was first introduced)

$$
\bar{X} \times_{0} \bar{X}=\left[\bar{X} \times \bar{X} ; \Delta_{\partial}\right], \quad \Delta_{\partial}:=\{(m, m) \in \partial \bar{X} \times \partial \bar{X}\}
$$

obtained by blowing-up the diagonal $\Delta_{\partial}$ in the corner, the blow-down map is denoted by $\beta: \bar{X} \times_{0} \bar{X} \rightarrow \bar{X} \times \bar{X}$. This is a smooth manifold with corners which has 3 boundary hypersurfaces: the front face ff obtained from blowing-up $\Delta_{\partial}$, and the right and left boundaries $\mathrm{rb}$ and lb which respectively project down to $\bar{X} \times \partial \bar{X}$ and $\partial \bar{X} \times \bar{X}$ under $\beta$. One can similarly define the blow-ups

$$
\bar{X} \times_{0} \partial \bar{X}:=\left[\bar{X} \times \partial \bar{X} ; \Delta_{\partial}\right], \quad \partial \bar{X} \times_{0} \bar{X}:=\left[\partial \bar{X} \times \bar{X} ; \Delta_{\partial}\right]
$$

which are manifolds with 1 corner of codimension 2 and 2 boundary hypersurfaces: the front face ff obtained from the blow-up and the left boundary lb which projects to $\partial \bar{X} \times \partial \bar{X}$ for $\bar{X} \times_{0} \partial \bar{X}$, respectively the front face $\mathrm{ff}$ and right boundary $\mathrm{rb}$ for $\partial \bar{X} \times_{0} \bar{X}$. We call $\beta_{l}, \beta_{r}$ the blow-down maps of (13) and we let $\rho_{\mathrm{ff}}, \rho_{\mathrm{lb}}$ and $\rho_{\mathrm{rb}}$ be boundary defining functions of these 
hypersurfaces in each case. Notice that the two spaces in (13) are canonically diffeomorphic to the submanifolds $\left\{\rho_{\mathrm{rb}}=0\right\} \subset \bar{X} \times_{0} \bar{X}$ and $\left\{\rho_{\mathrm{lb}}=0\right\} \subset \bar{X} \times_{0} \bar{X}$. Like in Section 3.2 in [24], the bundle ${ }^{0} \Sigma \nabla^{0} \Sigma^{*}$ lifts smoothly to these 3 blown-up manifolds through $\beta, \beta_{l}$ and $\beta_{r}$, we will use the notation

$$
\mathcal{E}:=\beta^{*}\left({ }^{0} \Sigma \nabla^{0} \Sigma^{*}\right), \quad \quad \varepsilon_{j}:=\beta_{j}^{*}\left({ }^{0} \Sigma \nabla^{0} \Sigma^{*}\right) \text { for } j=l, r
$$

for these bundles. The interior diagonal in $X \times X$ lifts to a submanifold $\Delta_{\iota}$ in $\bar{X} \times_{0} \bar{X}$ which intersects the boundary only at the front face (and does so transversally). Then it follows from [24, Prop 3.2] that the resolvent $R_{ \pm}(\lambda)$ has a Schwartz kernel $R_{ \pm}\left(\lambda ; m, m^{\prime}\right) \in C^{-\infty}(\bar{X} \times \bar{X} ; \mathcal{E})$ which lifts to $\bar{X} \times_{0} \bar{X}$ to a polyhomogeneous conormal distribution on $\bar{X} \times_{0} \bar{X} \backslash \Delta_{\iota}$

$$
\beta^{*} R_{ \pm}(\lambda) \in\left(\rho_{\mathrm{rb}} \rho_{\mathrm{lb}}\right)^{\lambda+\frac{n}{2}} C^{\infty}\left(\bar{X} \times_{0} \bar{X} \backslash \Delta_{\iota} ; \mathcal{E}\right) .
$$

Combined with Theorem 11, this structure result on $R_{ \pm}(\lambda)$ implies

Corollary 13. The Schwartz kernel of the Calderón projector $P_{\overline{\mathcal{H}}_{\partial}}$ associated to the Dirac operator has an asymptotic expansion in polar coordinates around the diagonal without log terms. In particular, the Wodzicki-Guillemin local residue density of $P_{\overline{\mathcal{H}}_{\partial}}$ vanishes.

Proof. Using Theorem 11, it suffices to show that $S(0)$ has this property. From [24, eq (4.10), Sec. 3], the kernel of $S(\lambda)$ is given outside the diagonal by

$$
S\left(\lambda ; y, y^{\prime}\right)=i\left[\left.\left(x x^{\prime}\right)^{-\lambda-\frac{n}{2}} R_{+}\left(\lambda ; x, y, x^{\prime}, y^{\prime}\right)\right|_{x=x^{\prime}=0}-\left.\left(x x^{\prime}\right)^{-\lambda-\frac{n}{2}} R_{-}\left(\lambda ; x, y, x^{\prime}, y^{\prime}\right)\right|_{x=x^{\prime}=0}\right]
$$

Since a boundary defining function $x^{\prime}$ of $\bar{X} \times \partial \bar{X}$ in $\bar{X} \times \bar{X}$ lifts to $\beta^{*} x^{\prime}=\rho_{\mathrm{rb}} \rho_{\mathrm{ff}} F$ for some $F>0$ smooth on $\bar{X} \times_{0} \bar{X}$ (and similarly $\beta^{*} x=\rho_{\mathrm{lb}} \rho_{\mathrm{ff}} F$ for some smooth $F>0$ ), one can use (14) to obtain

$$
\beta^{*}\left(\left(x x^{\prime}\right)^{-\lambda-\frac{n}{2}} R_{ \pm}(\lambda) \in \rho_{\mathrm{ff}}^{-2 \lambda-n} C^{\infty}\left(\bar{X} \times_{0} \bar{X} ; \mathcal{E}\right) .\right.
$$

Restricting to $x=x^{\prime}=0, y \neq y^{\prime}$ corresponds to restricting to the corner $\mathrm{lb} \cap \mathrm{rb}$ which is cannonically diffeomorphic to $M \times_{0} M=\left[M \times M ; \Delta_{\partial}\right]$ and thus the pull-back $\beta_{\partial}^{*} S(\lambda)$ of the kernel of $S(\lambda)$ has an expansion in polar coordinates at $\Delta_{\partial}$ with no log terms after setting $\lambda=0$.

From (9), we deduce that the kernel $E\left(\lambda ; m, y^{\prime}\right)$ of $E(\lambda)$ lifts to

$$
\beta_{l}^{*} E(\lambda) \in \rho_{\mathrm{lb}}^{\lambda+\frac{n}{2}} \rho_{\mathrm{ff}}^{-\lambda-\frac{n}{2}} C^{\infty}\left(\bar{X} \times_{0} \partial \bar{X} ; \mathcal{E}_{l}\right)
$$

where we used the identification between $\left\{\rho_{\mathrm{rb}}=0\right\} \subset \bar{X} \times_{0} \bar{X}$ and $\bar{X} \times_{0} \partial \bar{X}$. Here, obviously, this is the kernel of the operator acting from $L^{2}\left(M,{ }^{0} \Sigma ; \mathrm{dv}_{h_{0}}\right)$ to $L^{2}\left(X,{ }^{0} \Sigma ; \mathrm{dv}_{g}\right)$. We have a similar description

$$
\beta_{r}^{*} E^{*}(\lambda) \in \rho_{\mathrm{rb}}^{\lambda+\frac{n}{2}} \rho_{\mathrm{ff}}^{-\lambda-\frac{n}{2}} C^{\infty}\left(\partial \bar{X} \times_{0} \bar{X} ; \mathcal{E}_{r}\right) .
$$

So we deduce that the Schwartz kernel $K^{*}\left(y, x^{\prime}, y^{\prime}\right) \in C^{\infty}\left(\partial \bar{X} \times \bar{X} ;{ }^{0} \Sigma \nabla^{0} \Sigma^{*}\right)$ of $K^{*}$ with respect to the density $\left|\mathrm{dv}_{h_{0}} \otimes \mathrm{dv}_{\bar{g}}\right|=x^{n+1}\left|\mathrm{dv}_{h_{0}} \otimes \mathrm{dv}_{g}\right|$ lifts through $\beta_{r}$ to

$$
\beta_{r}^{*} K^{*}=\frac{1}{2} \beta_{r}^{*}\left(x^{-\frac{n}{2}} E^{*}(0)\right) \in \rho_{\mathrm{ff}}^{-n} C^{\infty}\left(\partial \bar{X} \times_{0} \bar{X} ; \mathcal{E}_{r}\right) .
$$

Similarly, for $K$ we have

$$
\beta_{l}^{*} K \in \rho_{\mathrm{ff}}^{-n} C^{\infty}\left(\bar{X} \times_{0} \partial \bar{X} ; \mathcal{E}_{l}\right) .
$$

When it is clear, we may omit ${ }^{0} \Sigma$ in the notations $L^{2}\left(\bar{X},{ }^{0} \Sigma, \operatorname{dv}_{g}\right), L^{2}\left(\partial X,{ }^{0} \Sigma, \mathrm{dv}_{h_{0}}\right)$ for simplicity. Now we have 
Lemma 14. The operator $K$ is bounded from $L^{2}\left(\partial \bar{X}, \mathrm{dv}_{h_{0}}\right)$ to $L^{2}\left(\bar{X}, \mathrm{dv}_{\bar{g}}\right)$, and so is its adjoint $K^{*}$ from $L^{2}\left(\bar{X}, \mathrm{dv}_{\bar{g}}\right)$ to $L^{2}\left(\partial \bar{X}, \mathrm{dv}_{h_{0}}\right)$. The range of $K^{*}$ acting on $L^{2}\left(\bar{X}, \mathrm{dv}_{\bar{g}}\right)$ is contained in $\overline{\mathcal{H}}_{\partial}$ and the kernel of $K$ contains $\overline{\mathcal{H}}_{\partial}^{\perp}$.

Proof. It is shown in Lemma 4.7 of [24] the following identity

$$
R_{+}(0)-R_{-}(0)=-\frac{i}{2}\left(E_{+}(0) E_{+}(0)^{*}+E_{-}(0) E_{-}(0)^{*}\right)=-\frac{i}{2} E(0) E(0)^{*}
$$

as operators from $\dot{C}^{\infty}\left(\bar{X},{ }^{0} \Sigma\right)$ to $x^{\frac{n}{2}} C^{\infty}\left(\bar{X},{ }^{0} \Sigma\right)$, so in particular this implies that

$$
K K^{*}=\frac{1}{2} i x^{-\frac{n}{2}}\left(R_{+}(0)-R_{-}(0)\right) x^{\frac{n}{2}+1}
$$

as operators. Using the isometry $\psi \rightarrow x^{-(n+1) / 2} \psi$ from $L^{2}\left(X, \mathrm{dv}_{g}\right)$ to $L^{2}\left(X, \mathrm{dv}_{\bar{g}}\right)$, we see that the operator $K K^{*}$ is bounded on $L^{2}\left(\bar{X}, \operatorname{dvol}_{\bar{g}}\right)$ if and only if $x^{\frac{1}{2}}\left(R_{+}(0)-R_{-}(0)\right) x^{\frac{1}{2}}$ is bounded on $L^{2}\left(X, \mathrm{dv}_{g}\right)$. Now by (14), the Schwartz kernel of $x^{\frac{1}{2}} R_{ \pm}(0) x^{\prime \frac{1}{2}}$ lifts on the blown-up space $\bar{X} \times_{0} \bar{X}$ as a conormal function

$$
\beta^{*}\left(x^{\frac{1}{2}} R_{ \pm}(0) x^{\prime \frac{1}{2}}\right) \in \rho_{\mathrm{lb}}^{\frac{n+1}{2}} \rho_{\mathrm{rb}}^{\frac{n+1}{2}} \rho_{\mathrm{ff}} C^{\infty}\left(\bar{X} \times_{0} \bar{X} ; \mathcal{E}\right)
$$

since $\left(x x^{\prime}\right)^{\frac{1}{2}}$ lifts to $\bar{X} \times_{0} \bar{X}$ to $\left(\rho_{\mathrm{rb}} \rho_{\mathrm{lb}}\right)^{\frac{1}{2}} \rho_{\mathrm{ff}} F$ for some $F>0$ smooth on $\bar{X} \times_{0} \bar{X}$. We may then use Theorem 3.25 of Mazzeo [33] to conclude that it is bounded on $L^{2}\left(X, \mathrm{dv}_{g}\right)$, and it is even compact according to Proposition 3.29 of [33]. As a conclusion, $K^{*}$ is bounded from $L^{2}\left(X, \mathrm{dv}_{\bar{g}}\right)$ to $L^{2}\left(\partial \bar{X}, \mathrm{dv}_{h_{0}}\right)$ and $K$ is bounded on the dual spaces. The fact that the range of $K^{*}$ is contained in $\overline{\mathcal{H}}_{\partial}$ comes directly from a density argument and the fact that for all $\psi \in$ $\dot{C}^{\infty}\left(\bar{X} ;{ }^{0} \Sigma\right), K^{*} \psi=-\left.\frac{1}{2} i\left[x^{-\frac{n}{2}}\left(R_{+}(0)-R_{-}(0)\right)\left(x^{\frac{n}{2}+1} \psi\right)\right]\right|_{\partial \bar{X}}$, and $x^{-\frac{n}{2}}\left(R_{+}(0)-R_{-}(0)\right)\left(x^{\frac{n}{2}+1} \psi\right)$ is a smooth harmonic spinor of $D_{\bar{g}}$ on $\bar{X}$.

The operator $K^{*} K$ acts on $L^{2}\left(\partial \bar{X}, \operatorname{dv}_{h_{0}}\right)$ as a compact operator, we actually obtain

Lemma 15. The operator $K^{*} K$ is a classical pseudo-differential operator of order -1 on $\partial \bar{X}$ and its principal symbol is given by

$$
\sigma_{\mathrm{pr}}\left(K^{*} K\right)(y ; \mu)=\frac{1}{4}|\mu|_{h_{0}}^{-1}\left(\operatorname{Id}+i \operatorname{cl}(\nu) \operatorname{cl}\left(\frac{\mu}{|\mu|_{h_{0}}}\right)\right)
$$

Proof. According to (16) and Lemma 28, the operator $K=\frac{1}{2} x^{-\frac{n}{2}} E(0)$ is a log-free classical pseudodifferential operator in the class $I_{\mathrm{lf}}^{-1}(\bar{X} \times M ; \mathcal{E})$ in the terminology of Subsection B.2, while $K^{*}$ is in the class $I_{\mathrm{lf}}^{-1}(M \times \bar{X} \mathcal{E})$. We can therefore apply Proposition 30 to deduce that $K^{*} K \in \Psi^{-1}(M ; \mathcal{E})$ is a classical pseudo-differential operator of order -1 on $M$. Moreover, from Proposition 30, the principal symbol is given by

$$
\sigma_{K^{*} K}(y, \mu)=(2 \pi)^{-2} \int_{0}^{\infty} \hat{\sigma}_{K^{*}}(y ;-x, \mu) . \hat{\sigma}_{K}(y ; x, \mu) d x
$$

where hat denotes Fourier transform in the variable $\xi$ and $\sigma_{K^{*}}(y, \xi, \mu), \sigma_{K}(y ; \xi, \mu)$ are the principal symbols of $K^{*}, K$. We have to compute for $|\mu|$ large the integral above. We know from [24] that the leading asymptotic in polar coordinates around $\Delta_{\partial}$ (or equivalently the normal operator at the front face) of $K=\frac{1}{2} x^{-n / 2} E(0)$ at the submanifold $\Delta_{\partial}$ is given in local coordinates by

$$
K(x, y, y+z) \sim \frac{1}{2} \pi^{-\frac{n+1}{2}} \Gamma\left(\frac{n+1}{2}\right) \rho^{-n-1}(x+\operatorname{cl}(\nu) \operatorname{cl}(z))
$$


where $\rho:=\left(x^{2}+|z|^{2}\right)^{1 / 2}$ is the defining function for the front face of $\bar{X} \times_{0} M$. To obtain the symbol, we need to compute the inverse Fourier transform in $(x, z)$ variables of the homogeneous distribution $\rho^{-n-1}(x+\operatorname{cl}(\nu) \operatorname{cl}(z))$. To do this, we use the analytic family of $L^{1}$ tempered distributions $\omega(\lambda)=\rho^{-n-1+\lambda}$ for $\Re(\lambda)>0$. We have

$$
\mathcal{F}_{(x, z) \rightarrow(\xi, \mu)}(\omega(\lambda))=(2 \pi)^{\frac{n+1}{2}} 2^{\lambda-\frac{n+1}{2}} \frac{\Gamma\left(\frac{\lambda}{2}\right)}{\Gamma\left(\frac{n+1-\lambda}{2}\right)} R^{-\lambda}
$$

for $R:=|(\xi, \mu)|$. This allows us to compute $\mathcal{F}(x \omega(\lambda))$ and $\mathcal{F}\left(z_{j} \omega(\lambda)\right)$, which turn out to be regular at $\lambda=0$. Thus by setting $\lambda=0$ we get after a short computation

$$
\sigma_{K}(y ; \xi, \mu)=i\left(\xi^{2}+|\mu|^{2}\right)^{-1}(\xi+\operatorname{cl}(\nu) \operatorname{cl}(\mu)) .
$$

This gives $\sigma_{K^{*}}\left(y, \xi^{\prime}, \mu\right)=-i\left(\left(\xi^{\prime}\right)^{2}+|\mu|^{2}\right)^{-1}\left(\xi^{\prime}-\operatorname{cl}(\nu) \operatorname{cl}(\mu)\right)$. Use the fact that the Fourier transform of the Heaviside function is $\pi \delta-\frac{i}{\xi}$. Then

$$
4 \sigma_{K^{*} K}(y ; \mu)=\pi^{-1} \int_{\mathbb{R}} R^{-2} d \xi-\pi^{-2} i \int_{\mathbb{R}^{2}}\left(R R^{\prime}\right)^{-2}\left(\xi \xi^{\prime}+|\mu|^{2}+\left(\xi^{\prime}-\xi\right) \operatorname{cl}(\nu) \operatorname{cl}(\mu)\right) \frac{d \xi d \xi^{\prime}}{\xi-\xi^{\prime}}
$$

in the sense of principal value for $\left(\xi-\xi^{\prime}\right)^{-1}$. The first term gives $|\mu|^{-1}$. In the second term, by symmetry in $\xi, \xi^{\prime}$, only the term $\pi^{-2} i \operatorname{cl}(\nu) \operatorname{cl}(\mu) \int_{\mathbb{R}^{2}}\left(R R^{\prime}\right)^{-2} d \xi d \xi^{\prime}$ contributes, and it gives $i|\mu|^{-2} \operatorname{cl}(\nu) \operatorname{cl}(\mu)$. This ends the proof.

In fact, we could also compute the principal symbol using the push-forward approach but the computation is slightly more technical.

We deduce easily from the two last lemmas

Corollary 16. There exists a pseudo-differential operator of order 1 on $\partial \bar{X}$, denoted $\left(K^{*} K\right)^{-1}$ such that $\left(K^{*} K\right)^{-1} K^{*} K=\mathcal{C}$.

Proof. Using Lemmas 14, 15, we deduce that if $D_{h_{0}}$ is the Dirac operator on the boundary $\partial \bar{X}$ equipped with the metric $h_{0}=\left.\bar{g}\right|_{T \partial \bar{X}}$, then $A:=4 K^{*} K+(\operatorname{Id}-\mathcal{C})\left(\operatorname{Id}+D_{h_{0}}^{2}\right)^{-1 / 2}(\operatorname{Id}-\mathcal{C})$ is a pseudo-differential operator of order -1 , thus compact, and with $\operatorname{ker} A=0$. Moreover, by Lemma $15,\left(1+D_{h_{0}}^{2}\right)^{\frac{1}{2}} A$ is classical pseudo-differential of order 0 with principal symbol equal to Id. This implies that $A$ is Fredholm and injective, thus invertible on $L^{2}$ with inverse denoted by $B$ and by standard pseudo-differential method $B$ is also classical pseudo-differential or order 0 . Let us define $\left(K^{*} K\right)^{-1}:=4 B\left(1+D_{h_{0}}^{2}\right)^{\frac{1}{2}}$, which is classical pseudo-differential of order 1 , then one has $\left(K^{*} K\right)^{-1} A=4 \mathrm{Id}$, and multplying this identity by $\mathcal{C}$ on the right, we obtain the claim.

3.3. The orthogonal projector on harmonic spinors on $\bar{X}$. We will construct and analyze the projector on the $L^{2}\left(\bar{X}, \mathrm{dv}_{\bar{g}}\right)$-closure $\overline{\mathcal{H}}\left(D_{\bar{g}}\right)$ of

$$
\mathcal{H}\left(D_{\bar{g}}\right):=\left\{\psi \in C^{\infty}\left(\bar{X} ;{ }^{0} \Sigma\right) ; D_{\bar{g}} \psi=0\right\} .
$$

For this, let us now define the operator

$$
P:=K\left(K^{*} K\right)^{-1} K^{*}
$$

which maps continuously $\dot{C}^{\infty}\left(\bar{X} ;{ }^{0} \Sigma\right)$ to $C^{\infty}\left(\bar{X} ;{ }^{0} \Sigma\right)$. Since $K$ is bounded on $L^{2}\left(\partial \bar{X}, \mathrm{dv}_{h_{0}}\right)$, Lemma 14 and Corollary 16 imply easily the following

Corollary 17. The operator $P$ satisfies $P K=K \mathcal{C}=K$ on $L^{2}\left(\partial \bar{X}, \mathrm{dv}_{h_{0}}\right)$. 
We want to show that $P$ extends to a bounded operator on $L^{2}\left(\bar{X}, \operatorname{dv}_{\bar{g}}\right)$ and study the structure of its Schwartz kernel. We first use the following composition result which is a consequence of Melrose's push-forward theorem [35]. The definition of polyhomogeneous functions and index sets is recalled in Appendix A.

Theorem 18. The operator $P:=K\left(K^{*} K\right)^{-1} K^{*}$ has a Schwartz kernel in $C^{-\infty}\left(\bar{X} \times \bar{X} ;\left({ }^{0} \Sigma \nabla\right.\right.$ $\left.\left.{ }^{0} \Sigma^{*}\right) \otimes \Omega^{\frac{1}{2}}\right)$ on $\bar{X} \times \bar{X}$ which lifts to $\bar{X} \times_{0} \bar{X}$ through $\beta$ to $k_{P} \beta^{*}\left(\left|\operatorname{dv}_{\bar{g}} \otimes \mathrm{dv}_{\bar{g}}\right|^{\frac{1}{2}}\right)$ with

$$
k_{P} \in \mathcal{A}_{\mathrm{phg}}^{J_{\mathrm{ff}}, J_{\mathrm{rb}}, J_{\mathrm{lb}}}\left(\bar{X} \times_{0} \bar{X} ; \mathcal{E}\right), \quad J_{\mathrm{ff}}=-(n+1) \cup(-2,1) \cup(0,3), \quad J_{\mathrm{rb}}=J_{\mathrm{lb}}=0
$$

where $\left|\mathrm{dv}_{\bar{g}} \otimes \mathrm{dv}_{\bar{g}}\right|$ is the Riemannian density trivializing $\Omega(\bar{X} \times \bar{X})$ induced by $\bar{g}$.

Proof. We start by composing $A \circ B$ where $A:=\left(K^{*} K\right)^{-1}\left(\operatorname{Id}+D_{h_{0}}^{2}\right)^{-1}$ and $B:=\left(\operatorname{Id}+D_{h_{0}}^{2}\right) K^{*}$. From Corollary 16, we know that $A$ is a classical pseudo-differential operator on $M$ of order -1 , so its kernel lifts to $M \times_{0} M$ as a polyhomogeneous conormal kernel and its index set (as a $b$-half-density) $E$ is of the form $-\frac{n}{2}+1+\mathbb{N}_{0} \cup\left(\frac{n}{2}+\mathbb{N}_{0}, 1\right)$. Now, since the lift of vector fields on $M$ by the b-fibration $M \times_{0} \bar{X} \rightarrow M \times \bar{X} \rightarrow M$ is smooth, tangent to the right boundary in $M \times_{0} \bar{X}$ and transverse to the front face $\mathrm{ff}$, we deduce that applying $\mathrm{Id}+D_{h_{0}}^{2}$ to $K^{*}$ reduces its order at ff by 2 and leaves the index set at rb invariant, so $\left(\mathrm{Id}+D_{h_{0}}^{2}\right) K^{*}$ has a kernel which lifts on $M \times_{0} \bar{X}$ to an element in $\mathcal{A}_{\mathrm{phg}}^{F_{\mathrm{f}}, F_{\mathrm{rb}}}\left(M \times_{0} \bar{X} ; \mathcal{E}_{r} \otimes \Omega_{b}^{\frac{1}{2}}\right)$ with

$$
F_{\text {ff }}=-\frac{n}{2}-\frac{3}{2}, \quad F_{\text {rb }}=\frac{1}{2} .
$$

So using Lemma 29, we deduce that $A \circ B$ has a kernel which lifts to $M \times_{0} \bar{X}$ as an element in

$$
\mathcal{A}_{\mathrm{phg}}^{H_{\mathrm{ff}}, H_{\mathrm{rb}}}\left(M \times_{0} \bar{X} ; \mathcal{E}_{r} \otimes \Omega_{b}^{\frac{1}{2}}\right), \quad H_{\mathrm{ff}} \subset\left(-\frac{n}{2}-\frac{1}{2}\right) \cup\left(\frac{n}{2}-\frac{3}{2}, 1\right) \cup\left(\frac{n}{2}+\frac{1}{2}, 2\right), \quad H_{\mathrm{rb}}=-\frac{3}{2} \overline{\frac{1}{2}}
$$

The index set $H_{\mathrm{rb}}$ must in fact be $\frac{1}{2}$ since the dual of this composition maps $C^{\infty}\left(M{ }^{0} \Sigma\right)$ into $C^{\infty}\left(\bar{X} ;{ }^{0} \Sigma\right)$ (with respect to the density $\left|\operatorname{dv}_{\bar{g}}\right|^{\frac{1}{2}}$ ). Now the operator $K$ has a kernel lifted to $\bar{X} \times_{0} M$ which is in $\rho_{\mathrm{ff}}^{-\frac{n}{2}+\frac{1}{2}} \rho_{\mathrm{lb}}^{\frac{1}{2}} C^{\infty}\left(\bar{X} \times_{0} M ; \mathcal{E}_{l} \otimes \Omega_{b}^{\frac{1}{2}}\right)$ thus using Lemma 29 (and the same argument as above to show that the index set is $\frac{1}{2}$ at $\mathrm{lb}, \mathrm{rb}$ ), we deduce that the lift $k_{P}$ of the Schwartz kernel of $P$ is polyhomogeneous conormal on $\bar{X} \times_{0} \bar{X}$, and the index set of $k_{P}$ satisfies (as a b-half-density)

$$
J_{\mathrm{ff}}=-\frac{n}{2} \cup\left(\frac{n}{2}-1,1\right) \cup\left(\frac{n}{2}+1,3\right), \quad J_{\mathrm{lb}}=J_{\mathrm{rb}}=\frac{1}{2} .
$$

Now this completes the proof since the lift of the half-density $\left|\mathrm{dv}_{\bar{g}} \otimes \mathrm{dv}_{\bar{g}}\right|^{\frac{1}{2}}$ is of the form $\rho_{\mathrm{ff}}^{\frac{n}{2}+1} \rho_{\mathrm{lb}}^{\frac{1}{2}} \rho_{\mathrm{rb}}^{\frac{1}{2}} \mu_{b}^{\frac{1}{2}}$ where $\mu_{b}$ is a non vanishing smooth section of $\Omega_{b}$.

Corollary 19. The operator $P=K\left(K^{*} K\right)^{-1} K^{*}$ is bounded on $L^{2}\left(\bar{X}, \operatorname{dv}_{\bar{g}}\right)$ and is the orthogonal projector on the $L^{2}$-closure of the set of smooth harmonic spinors for $D_{\bar{g}}$ on $\bar{X}$, that is, $P=P_{\overline{\mathcal{H}}}$.

Proof. Let $P^{\prime}:=x^{\frac{n+1}{2}} P x^{-\frac{n+1}{2}}$ acting on $\dot{C}^{\infty}\left(\bar{X} ;{ }^{0} \Sigma\right)$, then it suffices to prove that $P^{\prime}$ extends to a bounded operator on $L^{2}\left(X, \mathrm{dv}_{g}\right)$. But, in terms of half-densities, the half density $\mid \mathrm{dv}_{g} \otimes$ 
$\left.\mathrm{dv}_{g}\right|^{\frac{1}{2}}$ is given by $\left(x x^{\prime}\right)^{-\frac{n+1}{2}}\left|\mathrm{dv}_{\bar{g}} \otimes \mathrm{dv}_{\bar{g}}\right|^{\frac{1}{2}}$ and Theorem 18 shows that the Schwartz kernel of $P^{\prime}$ lifts on $\bar{X} \times_{0} \bar{X}$ to a half-density $k_{P^{\prime}} \beta^{*}\left(\left|\mathrm{dv}_{g} \otimes \mathrm{dv}_{g}\right|^{\frac{1}{2}}\right)$ where

$$
k_{P^{\prime}} \in \mathcal{A}_{\mathrm{phg}}^{J_{\mathrm{ff}}^{\prime}, J_{\mathrm{rb}}^{\prime}, J_{\mathrm{lb}}^{\prime}}\left(\bar{X} \times_{0} \bar{X} ; \varepsilon\right) \quad J_{\mathrm{ff}}^{\prime} \geq 0, \quad J_{\mathrm{rb}}^{\prime}=J_{\mathrm{lb}}^{\prime}=\frac{n+1}{2} .
$$

It is proved in Proposition 3.20 of Mazzeo [33] that such operators are bounded on $L^{2}\left(\bar{X}, \mathrm{dv}_{g}\right)$. To conclude, we know from Corollary 17 that $P$ is the identity on the range of $K$ acting on $C^{\infty}\left(\partial \bar{X} ;{ }^{0} \Sigma\right)$, which coincides with the space of smooth harmonic spinors for $D_{\bar{g}}$ on $\bar{X}$, and we also know that $P$ vanishes on $\operatorname{ker}\left(K^{*}\right)=\overline{\operatorname{Im}(K)}^{\perp}$, so this achieves the proof.

\section{Conformally covariant powers of Dirac operators and Cobordism INVARIANCE OF THE INDEX}

In this section, we define some conformally covariant differential operators with leading part given by a power of the Dirac operator. The method is the same as in Graham-Zworski [20], using our construction of the scattering operator in Section 2. Since this is very similar to the case of functions dealt with in [20], we do not give much details. Let $(X, g)$ be an asymptotically hyperbolic manifold with a metric $g$, and let $x$ be a geodesic boundary defining function of $\partial \bar{X}$ so that the metric has a product decomposition of the form $g=\left(d x^{2}+h(x)\right) / x^{2}$ near $\partial \bar{X}$ as in (3).

Lemma 20. Let $C(\lambda):=2^{-2 \lambda} \Gamma(1 / 2-\lambda) / \Gamma(1 / 2+\lambda)$. If the metric $g$ is even to infinite order, the operator $\widetilde{S}(\lambda):=S(\lambda) / C(\lambda)$ is finite meromorphic in $\mathbb{C}$, and it is holomorphic in $\{\Re(\lambda) \geq 0\}$. Moreover for $k \in \mathbb{N}_{0}$, the operator $L_{k}:=\widetilde{S}(1 / 2+k)$ is a conformally covariant self-adjoint differential operator on $\partial \bar{X}$ with leading part $\operatorname{cl}(\nu) D_{h_{0}}^{1+2 k}$, and it depends only on the tensors $\partial_{x}^{2 j} h(0)$ in a natural way for $j \leq k$. For $k=0$, one has $\widetilde{S}(1 / 2)=\operatorname{cl}(\nu) D_{h_{0}}$.

Proof. The first statement is proved in Corollary 4.11 of [24]. The last statement about $\widetilde{S}(1 / 2+k)$ is a consequence of the construction of $\sigma_{ \pm}(\lambda)$ in Proposition 6 , by copying mutatis mutandis the proof of Theorem 1 of Graham-Zworski [20]. Indeed, by construction, the term $\sigma_{\infty, \pm}$ satisfying (6) has a Taylor expansion at $x=0$ of the form

$$
\sigma_{\infty, \pm}(\lambda)=\psi+\sum_{j=1}^{k} x^{j}\left(p_{j, \lambda} \psi\right)+O\left(x^{k+1}\right)
$$

for all $k \in \mathbb{N}$ where $p_{j, \lambda}$ are differential operators acting on $C^{\infty}\left(\partial \bar{X},{ }^{0} \Sigma\right)$ such that $\frac{p_{j, \lambda}}{\Gamma(1 / 2-\lambda)}$ are holomorphic in $\{\Re(\lambda) \geq 0\}$ and depend in a natural way only on the tensors $\left(\partial_{x}^{\ell} h(0)\right)_{\ell \leq j}$. Following Proposition 3.5 and Proposition 3.6 in [20], the operator $\operatorname{Res}_{\lambda=1 / 2+k} S(\lambda)$ is also equal to $-\operatorname{Res}_{\lambda=1 / 2+k}\left(p_{2 k+1, \lambda}\right)$. The computation of $\widetilde{S}(1 / 2)$ is then rather straightforward by checking that

$$
p_{1, \lambda}=-\frac{\operatorname{cl}(\nu) D_{h_{0}}}{2 \lambda-1}
$$

using the indicial equation (8) and the decomposition (7).

A first corollary of Lemma 20 is the cobordism invariance of the index of the Dirac operator.

Corollary 21. Let $D_{h_{0}}$ be the Dirac operator on a $2 k$-dimensional closed spin manifold $\left(M, h_{0}\right)$ which is the oriented boundary of a compact manifold with boundary $(\bar{X}, \bar{g})$. Let $D_{h_{0}}^{+}$ 
be the restriction of $D_{h_{0}}$ to the sub-bundle of positive spinors $\Sigma^{+}:=\operatorname{ker}(\omega-1)$, where $\omega$ is the Clifford multiplication by the volume element when $k$ is even, respectively $\omega=i \mathrm{cl}\left(\operatorname{vol}_{h_{0}}\right)$ for $k$ odd. Then $\operatorname{Ind}\left(D_{h_{0}}^{+}\right)=0$.

Proof. By topological reasons, we may assume that $\bar{X}$ is also spin and that the spin structure on $M$ is induced from that on $\bar{X}$. Using the isomorphism between the usual spin bundle $\Sigma(X)$ and the 0 -spin bundle ${ }^{0} \Sigma(X)$ in Section 2 , we see that $D_{h_{0}}$ can be considered as acting in the restriction of the 0 -spin bundle ${ }^{0} \Sigma$ to $M$. Since the odd-dimensional spin representation is chosen such that $\operatorname{cl}(\nu)=i \omega$, the $\pm i$ eigenspaces of $\operatorname{cl}(\nu)$ on $\left.{ }^{0} \Sigma(X)\right|_{M}$ correspond to the splitting in positive, respectively negative spinors defined by $\omega$ on $\Sigma(M)$. We have seen that $\widetilde{S}(1 / 2)=\operatorname{cl}(\nu) D_{h_{0}}$. Then by the homotopy invariance of the index, it suffices to use the fact that $\widetilde{S}(\lambda)$ is invertible for all $\lambda$ except in a discrete set of $\mathbb{C}$, which follows from Lemma 20 and Proposition 7.

We refer for instance to $[1,38,30,41,8]$ for other proofs of the cobordism invariance of the index of $D^{+}$.

Now, let us consider $M$ a compact manifold equipped with a conformal class $\left[h_{0}\right]$. A $(n+1)$-dimensional Poincaré-Einstein manifold $(X, g)$ associated to $\left(M,\left[h_{0}\right]\right)$ is an asymptotically hyperbolic manifold with conformal infinity $\left(M,\left[h_{0}\right]\right)$ and such that the following extra condition holds near the boundary $M=\partial \bar{X}$

$$
\operatorname{Ric}(g)=-n g+O\left(x^{N-2}\right), \quad N= \begin{cases}\infty & \text { if } n+1 \text { is even, } \\ n & \text { if } n+1 \text { is odd }\end{cases}
$$

Notice that by considering the disjoint union $M_{2}:=M \sqcup M$ instead of $M$, one sees that either $M$ or $M_{2}$ can be realized as the boundary of a compact manifold with boundary $\bar{X}$.

Fefferman and Graham $[16,17]$ proved that for any $\left(M,\left[h_{0}\right]\right)$ which is the boundary of a compact manifold $\bar{X}$, there exist Poincaré-Einstein manifolds associated to $\left(M,\left[h_{0}\right]\right)$. Moreover writing $g=\left(d x^{2}+h(x)\right) / x^{2}$ for a geodesic boundary defining function $x$, the Taylor expansion of the metric $h(x)$ at $M=\{x=0\}$ is uniquely locally (and in a natural way) determined by $h_{0}=h(0)$ and the covariant derivatives of the curvature tensor of $h_{0}$, but not on the Poincaré-Einstein metrics associated to $\left(M,\left[h_{0}\right]\right)$. If $M$ is spin, we can always construct a Poincaré-Einstein $(X:=[0,1] \times M, g)$ associated to $M_{2}$ with a spin structure induced naturally by that of $M$.

Corollary 22. If $(X, g)$ is a spin Poincaré-Einstein manifold associated to a spin conformal manifold $\left(M,\left[h_{0}\right]\right)$, then for $k \leq N / 2$ the operators $L_{k}=:-\operatorname{cl}(\nu) \widetilde{S}(1 / 2+k)$ acting on $C^{\infty}\left(M,{ }^{0} \Sigma\right)$ are self-adjoint natural (with respect to $\left.h_{0}\right)$, conformally covariant differential operators of the form $L_{k}=D_{h_{0}}^{2 k+1}+$ lower order terms.

Hence we can then always define the operators $L_{k}$ on $M_{2}=M \sqcup M$ and thus, since the construction is local and natural with respect to $h_{0}$, this defines naturally $L_{k}$ on any $M$. As above, when $\left(M,\left[h_{0}\right]\right)$ is a boundary, the index of the restriction $L_{k}^{ \pm}$to ${ }^{0} \Sigma_{ \pm}=\operatorname{ker}(\omega \mp 1)$ (when $n$ is even) is always 0 . In general, the index of $L_{k}^{ \pm}$is the index of $L_{0}^{ \pm}$, which equals the $\hat{A}$-genus of $M$ by the Atiyah-Singer index theorem [1].

4.1. The Dirac operator. For $k=0$, the operator $L_{0}$, which is essentially the pole of the scattering matrix at $\lambda=1 / 2$, is just the Dirac operator $D_{h_{0}}$ on $\left(M, h_{0}\right)$ when the dimension of $M$ is even, respectively two copies of $D_{h_{0}}$ when $\operatorname{dim}(M)$ is odd. 
4.2. A conformally covariant operator of order 3. For $k=1$ in Corollary 22 we get a conformally covariant operator of order 3 on any spin manifold of dimension $n \geq 3$, with the same principal symbol as $D_{h_{0}}^{3}$.

Theorem 23. Let $\left(M, h_{0}\right)$ be a Riemannian spin manifold of dimension $n \geq 3$. Then the differential operator of order 3 acting on spinors

$$
L_{1}:=D_{h_{0}}^{3}-\frac{2 \mathrm{cl} \circ \operatorname{Ric}_{h_{0}} \circ \nabla^{h_{0}}}{n-2}+\frac{\mathrm{scal}_{h_{0}}}{(n-1)(n-2)} D_{h_{0}}-\frac{\mathrm{cl}\left(d\left(\mathrm{scal}_{h_{0}}\right)\right)}{2(n-1)}
$$

is conformally covariant with respect to $h_{0}$ in the following sense: if $\omega \in C^{\infty}(M)$ and $\hat{h}_{0}=$ $e^{2 \omega} h_{0}$, then

$$
\hat{L}_{1}=e^{-\frac{n+3}{2} \omega} L_{1} e^{\frac{n-3}{2} \omega}
$$

where $\hat{L}_{1}$ is defined as above but using the metric $\hat{h}_{0}$ instead of $h_{0}$.

Proof. The existence of the operator $L_{1}$ with the above covariance property is already established, we are now going to compute it explicitly. The asymptotic expansion of the PoincaréEinstein metric $g=x^{-2}\left(d x^{2}+h_{x}\right)$ at the boundary is given in [17] by

$$
\bar{g}=x^{2} g=d x^{2}+h_{0}-x^{2} P+O\left(x^{4}\right), \quad P=\frac{1}{n-2}\left(\operatorname{Ric}_{h_{0}}-\frac{\text { scal }_{h_{0}}}{2(n-1)}\right) .
$$

We trivialize the spinor bundle on $(\bar{X}, \bar{g})$ from the boundary using parallel transport along the gradient vector field $X:=\partial_{x}$. Let us write the limited Taylor series of $D_{\bar{g}}$ in this trivialization:

$$
D_{\bar{g}}=\operatorname{cl}(\nu) \partial_{x}+D_{0}+x D_{1}+x^{2} D_{2}+O\left(x^{3}\right) .
$$

Use the conformal change formula

$$
D_{g}=x^{\frac{n+2}{2}} D_{\bar{g}} x^{-\frac{n}{2}}
$$

valid in dimension $n+1$. The idea from [20] is to use the formal computation giving the residue of the scattering operator at $\lambda=\frac{3}{2}$ in terms of the $x^{\frac{n}{2}+3} \log (x)$ coefficient in the asmymptotic expansion of formal solution to $\left(D_{g}-\frac{3}{2} i\right) \omega=0$ (the same method has been used in [2] for forms): there is a unique solution $\omega$ modulo $O\left(x^{\frac{n}{2}+3}\right)$ of $\left(D_{g}-\frac{3}{2} i\right) \omega=O\left(x^{\frac{n}{2}+3}\right)$ of the form

$$
\omega=x^{\frac{n}{2}}\left(x^{-\frac{3}{2}} \omega_{0}^{-}+\sum_{j=1}^{2} x^{j-\frac{3}{2}} \omega_{j}^{ \pm}+x^{\frac{3}{2}} \log x \cdot \nu^{+}\right)+O\left(x^{\frac{n}{2}+3}\right)
$$

and $\nu^{+}=C_{k} \operatorname{Res}_{\lambda=3 / 2} S(\lambda) \omega_{0}^{-}=C_{k}^{\prime} \operatorname{cl}(\nu) L_{1}\left(\omega_{0}^{-}\right)$for some non-zero constants $C_{k}, C_{k}^{\prime}$. Since we know the principal term of $L_{1}$ is $D_{h_{0}}^{3}$, we can renormalize later and the constant $C_{k}^{\prime}$ is irrelevant in the computation. Recall that spinors in the $\pm i$ eigenspaces of $\operatorname{cl}(\nu)$ are denoted with a \pm symbol.

Lemma 24. The conformally covariant operator of order 3 from Corollary 22 is given on by

$$
L_{1}=D_{0}^{3}+2 \operatorname{cl}(\nu)\left(D_{1} D_{0}+D_{0} D_{1}\right)-4 D_{2} .
$$

Proof. From (19), (20) and (21) we derive by a straightforward computation the identity (22) on negative spinors. The same formula is obtained when we start with $\omega_{0}^{+}$, so the lemma is proved. 
Lemma 25. The operators $D_{1}, D_{2}$ are given by

$$
\begin{aligned}
D_{1} & =-\frac{\operatorname{scal}_{h_{0}} \operatorname{cl}(\nu)}{4(n-1)} \\
-4 D_{2} & =-2 \operatorname{cl} \circ P \circ \nabla=-\frac{2}{n-2} \sum_{i, j=1}^{n} \operatorname{cl}_{i} \operatorname{Ric}_{h_{0} ; i j} \nabla_{j}+\frac{2 \operatorname{scal}_{h_{0}} D_{0}}{2(n-1)(n-2)} .
\end{aligned}
$$

Proof. We write $\langle U, V\rangle$ for the scalar product with respect to the $\bar{g}$ metric, and $\nabla$ for the Riemannian connection. Notice that for $U, V$ vectors tangent to the $\left\{x=x_{0}\right\}$ slices, and for $A$ defined by the identity $P(U, V)=h_{0}(A U, V)$, we have

$$
\langle U, V\rangle=h_{0}\left(U-x^{2} A U, V\right)+O\left(x^{4}\right) .
$$

Let $U, V$ be local vector fields on $M$. We first extend them to be constant in the $x$ direction with respect to the product structure $(0, \epsilon)_{x} \times M$. Then

$$
\left\langle\nabla_{X} U, V\right\rangle=-x h_{0}(A U, V)+O\left(x^{3}\right)
$$

which implies that the vector field

$$
\tilde{U}:=U+\frac{x^{2}}{2} A U
$$

is parallel with respect to $X$ modulo $O\left(x^{3}\right)$. Let $\left(U_{j}\right)_{1 \leq j \leq n}$ be a local orthonormal frame on $M$. Then $\left(X, \tilde{U}_{1}, \ldots, \tilde{U}_{n}\right)$ is an orthonormal frame on $(0, \epsilon) \times M$ up to order $O\left(x^{4}\right)$ and parallel with respect to $X$ to order $O\left(x^{3}\right)$. To compute the Dirac operator of $\bar{g}$, we use the trivialization of the spinor bundle "from the boundary" given by the Gram-Schmidt orthonormalisation of this frame with respect to $\bar{g}$, which introduces an extra error term of order $O\left(x^{4}\right)$ (therefore harmless). Notice that

$$
[\tilde{U}, \tilde{V}]=\widetilde{[U, V]}-\frac{x^{2}}{2}(A[U, V]-[U, A V]-[A U, V]) .
$$

Then we compute from the Koszul formula

$$
\begin{gathered}
\nabla_{X} \tilde{U}_{j}=O\left(x^{3}\right), \quad \nabla_{X} X=0, \quad \nabla_{\tilde{U}_{j}} X=-x A \tilde{U}_{j}+O\left(x^{3}\right), \\
2\left\langle\nabla_{\tilde{U}_{j}} \tilde{U}_{i}, \tilde{U}_{k}\right\rangle=2 h_{0}\left(\nabla_{U_{j}}^{h_{0}} U_{i}, U_{k}\right)-\frac{x^{2}}{2} \\
\left\{h_{0}\left(A\left[U_{j}, U_{i}\right]-\left[U_{j}, A U_{i}\right]-\left[A U_{j}, U_{i}\right], U_{k}\right)\right. \\
+h_{0}\left(A\left[U_{k}, U_{j}\right]-\left[U_{k}, A U_{j}\right]-\left[A U_{k}, U_{j}\right], U_{i}\right) \\
\left.+h_{0}\left(A\left[U_{k}, U_{i}\right]-\left[U_{k}, A U_{i}\right]-\left[A U_{k}, U_{i}\right], U_{j}\right)\right\}+O\left(x^{3}\right) .
\end{gathered}
$$

We continue the computation at a point $p$ assuming that the frame $U_{j}$ is radially parallel from $p$, in particular at $p$ we have $\left(\nabla_{U_{j}}^{h_{0}} U_{i}\right)(p)=0,\left[U_{j}, U_{i}\right](p)=0$ and $U_{j}(p)=\partial_{j}$ i.e., at $p$ the vector fields $U_{j}$ are just the coordinate vectors of the geodesic normal coordinates. Then the coefficient of $\frac{x^{2}}{2}$ in $2\left\langle\nabla_{\tilde{U}_{j}} \tilde{U}_{i}, \tilde{U}_{k}\right\rangle$ simplifies a lot, and we get at $p$

$$
2\left\langle\nabla_{\tilde{U}_{j}} \tilde{U}_{i}, \tilde{U}_{k}\right\rangle=2 h_{0}\left(\nabla_{U_{j}}^{h_{0}} U_{i}, U_{k}\right)-x^{2}\left(\partial_{i} A_{k j}-\partial_{k} A_{i j}\right)+O\left(x^{3}\right) .
$$


From the local formula for the Dirac operator [4, Eq 3.13] we obtain

$$
\begin{aligned}
D_{\bar{g}}= & \operatorname{cl}(X) \partial_{x}+\operatorname{cl}_{j}\left(U_{j}+\frac{x^{2}}{2} A U_{j}\right)-\frac{1}{2} \sum_{j, k=1}^{n} x A_{j k} \operatorname{cl}_{j} \operatorname{cl}(X) \mathrm{cl}_{k}+\frac{1}{2} \sum_{i<k} h_{0}\left(\nabla_{U_{j}}^{h_{0}} U_{i}, U_{k}\right) \mathrm{cl}_{j} \mathrm{cl}_{i} \mathrm{cl}_{k} \\
& -\frac{x^{2}}{4} \sum_{j=1}^{n} \sum_{i<k}\left(\partial_{i} A_{k j}-\partial_{k} A_{i j}\right) \mathrm{cl}_{j} \operatorname{cl}_{i} \operatorname{cl}_{k}+O\left(x^{3}\right) .
\end{aligned}
$$

It follows that $D_{0}$ is just the Dirac operator for $h_{0}$. For $D_{1}$, we could additionally assume that at $p$, the vectors $U_{j}$ are eigenvectors of $A$, thus $D_{1}=\frac{1}{2} \operatorname{tr}_{h_{0}}(A) \operatorname{cl}(X)$ which in view of the definition of $P$ (recall that $A$ is the transformation corresponding to $P$ with respect to $h_{0}$ ) implies after a short computation the first formula of the lemma.

We also get

$$
D_{2}=\frac{1}{2} \operatorname{cl}_{j} A U_{j}-\frac{1}{4} \sum_{j=1}^{n} \sum_{i<k}\left(\partial_{i} A_{k j}-\partial_{k} A_{i j}\right) \mathrm{cl}_{j} \mathrm{cl}_{i} \mathrm{cl}_{k},
$$

but in the first term the action of $U_{j}$ at $p$ clearly coincides with the covariant derivative (the frame is parallel at $p$ ) so we get the advertised formula. As for the second term, it turns out to vanish miraculously because of the coefficients inside $P$. Indeed, due to the Clifford commutations we first check that the sum where $j, i, k$ are all distinct vanishes. The remaining sum is given at $p$ by

$$
\sum_{i, k} \operatorname{cl}_{k}\left(\partial_{k} A_{i i}-\partial_{i} A_{i k}\right)
$$

which in invariant terms reads

$$
\operatorname{cl}\left(d\left(\operatorname{tr}_{h_{0}}(A)\right)\right)+\operatorname{cl}\left(\delta^{\nabla}(A)\right)
$$

where $\delta^{\nabla}$ is the formal adjoint of the symmetrized covariant derivative with respect to $h_{0}$. It is known that

and from

$$
\delta^{\nabla} \operatorname{Ric}_{h_{0}}+\frac{d\left(\operatorname{scal}_{h_{0}}\right)}{2}=0
$$

$$
\operatorname{tr}_{h_{0}}\left(\operatorname{Ric}_{h_{0}}\right)=\operatorname{scal}_{h_{0}}, \quad \operatorname{tr}_{h_{0}}(A)=\frac{\operatorname{scal}_{h_{0}}}{2(n-1)}, \quad \delta^{\nabla}\left(\operatorname{scal}_{h_{0}} \cdot I\right)=-d\left(\operatorname{scal}_{h_{0}}\right)
$$

we get the result.

This lemma ends the proof of the theorem by using (22).

Appendix A. Polyhomogeneous conormal distributions, Densities, Blow-ups AND INDEX SETS

On a compact manifold with corners $\bar{X}$, consider the set of boundary hypersurfaces $\left(H_{j}\right)_{j=1}^{m}$ which are codimension 1 submanifolds with corners. Let $\rho_{1}, \ldots, \rho_{m}$ be some boundary defining functions of these hypersurfaces. An index set $\mathcal{E}=\left(\mathcal{E}_{1}, \ldots, \mathcal{E}_{m}\right)$ is a subset of $\left(\mathbb{C} \times \mathbb{N}_{0}\right)^{m}$ such that for each $M \in \mathbb{R}$ the number of points $(\beta, j) \in \mathcal{E}_{j}$ with $\Re(\beta) \leq M$ is finite, if $(\beta, k) \in \mathcal{E}_{j}$ then $(\beta+1, k) \in \mathcal{E}_{j}$, and if $k>0$ then also $(\beta, k-1) \in \mathcal{E}_{j}$. We define the set

$$
\dot{C}^{\infty}(\bar{X}):=\left\{f \in C^{\infty}(\bar{X}) ; f \text { vanishes to all orders on each } H_{j}\right\} \text {. }
$$

Its dual $C^{-\infty}(\bar{X})$ is called the set of extendible distributions (the duality pairing is taken with respect to a fixed smooth 1-density on $\bar{X}$ ). Conormal distributions on manifolds with 
corners were defined and analyzed by Melrose [35, 36], we refer the reader to these works for more details, but we give here some definitions. We say that an extendible distribution $f$ on a manifold with corners $X$ with boundary hypersurfaces $\left(H_{1}, \ldots, H_{m}\right)$ is polyhomogeneous conormal (phg for short) at the boundary, with index set $\mathcal{E}=\left(\varepsilon_{1}, \ldots, \varepsilon_{m}\right)$, if it is smooth in the interior $X$, conormal (i.e. if it remains in a fixed weighted $L^{2}$ space under repeated application of vector fields tangent to the boundary of $\bar{X}$ ) and if for each $s \in \mathbb{R}$ we have

$$
\left(\prod_{j=1}^{m} \prod_{\substack{(z, p) \in \mathcal{E}_{j} \\ \text { s.t. } \Re(z) \leq s}}\left(V_{j}-z\right)\right) f=O\left(\left(\prod_{j=1}^{m} \rho_{j}\right)^{s}\right)
$$

where $V_{j}$ is a smooth vector field on $\bar{X}$ that takes the form $V_{j}=\rho_{j} \partial_{\rho_{j}}+O\left(\rho_{j}^{2}\right)$ near $H_{j}$. This implies that $f$ has an asymptotic expansion in powers and logarithms near each boundary hypersurface. In particular, near the interior of $H_{j}$, we have

$$
f=\sum_{\substack{(z, p) \in \mathcal{E}_{j} \\ \text { s.t. } \Re(z) \leq s}} a_{(z, p)} \rho_{j}^{z}\left(\log \rho_{j}\right)^{p}+O\left(\rho_{j}^{s}\right)
$$

for every $s \in \mathbb{R}$, where $a_{(z, p)}$ is smooth in the interior of $H_{j}$, and $a_{(z, p)}$ is itself polyhomogeneous on $H_{j}$. The set of polyhomogeneous conormal distributions with index set $\mathcal{E}$ on $\bar{X}$ with values in a smooth bundle $F \rightarrow \bar{X}$ will be denoted by

$$
\mathcal{A}_{\mathrm{phg}}^{\mathcal{E}}(\bar{X} ; F) \text {. }
$$

Recall the operations of addition and extended union of two index sets $E_{1}$ and $E_{2}$, denoted by $E_{1}+E_{2}$ and $E_{1} \cup E_{2}$ respectively:

$$
\begin{aligned}
& E_{1}+E_{2}=\left\{\left(\beta_{1}+\beta_{2}, j_{1}+j_{2}\right) \mid\left(\beta_{1}, j_{1}\right) \in E_{1} \text { and }\left(\beta_{2}, j_{2}\right) \in E_{2}\right\} \\
& E_{1} \cup E_{2}=E_{1} \cup E_{2} \cup\left\{(\beta, j) \mid \exists\left(\beta, j_{1}\right) \in E_{1},\left(\beta, j_{2}\right) \in E_{2} \text { with } j=j_{1}+j_{2}+1\right\} .
\end{aligned}
$$

In what follows, we shall write $q$ for the index set $\{(q+n, 0) \mid n=0,1,2, \ldots\}$ for any $q \in \mathbb{R}$. For any index set $E$ and $q \in \mathbb{R}$, we write $E \geq q$ if $\Re(\beta) \geq q$ for all $(\beta, j) \in E$ and if $(\beta, j) \in E$ and $\Re(\beta)=q$ implies $j=0$. Finally we say that $E$ is integral if $(\beta, j) \in E$ implies that $\beta \in \mathbb{Z}$.

On $\bar{X}$, the most natural densities are the $b$-densities introduced by Melrose $[35,36]$. The bundle $\Omega_{b}(\bar{X})$ of $b$-densities is defined to be $\rho^{-1} \Omega(\bar{X})$ where $\rho=\prod_{j} \rho_{j}$ is a total boundary defining function and $\Omega(\bar{X})$ is simply the usual smooth bundle of densities on $\bar{X}$. In particular a smooth section of the $b$-densities bundle restricts canonically on each $H_{j}$ to a smooth $b$ density on $H_{j}$. The bundle of $b$-half-densities is simply $\rho^{-\frac{1}{2}} \Omega^{\frac{1}{2}}(\bar{X})$.

A natural class of submanifolds, called p-submanifolds, of manifolds with corners is defined in Definition 1.7.4 in [37]. If $Y$ is a closed $p$-submanifold of $\bar{X}$, one can define the blow-up $[\bar{X} ; Y]$ of $\bar{X}$ around $Y$, this is a smooth manifold with corners where $Y$ is replaced by its inward pointing spherical normal bundle $S^{+} N Y$ and a smooth structure is attached using polar coordinates around $Y$. The new boundary hypersurface is diffeomorphic to $S^{+} N Y$ and is called front face of $[\bar{X} ; Y]$, there is a canonical smooth blow-down map $\beta:[\bar{X} ; Y] \rightarrow \bar{X}$ which is the identity outside the front face and the projection $S^{+} N Y \rightarrow Y$ on the front face. See section 5.3 of [37] for details. The pull-back $\beta^{*}$ maps continuously $\dot{C}^{\infty}(\bar{X})$ to $\dot{C}^{\infty}([\bar{X} ; Y])$ and it is a one-to-one correspondence, giving by duality the same statement for extendible distributions. 
Appendix B. Compositions of Kernels CONORMAL to the BOUNDARY DiAgOnAL

In this section, we introduce a symbolic way to describe conormal distributions associated to the diagonal $\Delta_{\partial}$ inside the corner of $\bar{X} \times \bar{X}, \bar{X} \times \partial \bar{X}$, or $\partial \bar{X} \times \bar{X}$. In particular, we compare the class of operators introduced by Mazzeo-Melrose (the 0-calculus) to a natural class of pseudo-differential operators we define by using oscillatory integrals. We will prove composition results using both the push-forward Theorem of Melrose [35] and some classical symbolic calculus. We shall use the notations from the previous sections.

B.1. Operators on $\bar{X}$. We say that an operator $K: \dot{C}^{\infty}(\bar{X}) \rightarrow C^{-\infty}(\bar{X})$ is in the class $I^{s}\left(\bar{X} \times \bar{X}, \Delta_{\partial}\right)$ if its Schwartz kernel $K\left(m, m^{\prime}\right) \in C^{-\infty}(\bar{X} \times \bar{X})$ is the sum of a smooth function $K_{\infty} \in C^{\infty}(\bar{X} \times \bar{X})$ and a singular kernel $K_{s}$ supported near $\Delta_{\partial}$, which can be written in local coordinates $\left(x, y, x^{\prime}, y^{\prime}\right)$ near a point $\left(0, y_{0}, 0, y_{0}\right) \in \Delta_{\partial}$ under the form (here $x$ is a boundary defining function on $\bar{X}$ and $y$ some local coordinates on $\partial \bar{X}$ near $y_{0}$, and prime denotes the right variable version of them)

$$
K_{s}\left(x, y, x^{\prime}, y^{\prime}\right)=\frac{1}{(2 \pi)^{n+2}} \int_{\mathbb{R}} \int_{\mathbb{R}} \int_{\mathbb{R}^{n}} e^{-i x \xi-i x^{\prime} \xi^{\prime}-i\left(y-y^{\prime}\right) \mu} a\left(x, y, x^{\prime}, y^{\prime} ; \xi, \xi^{\prime}, \mu\right) d \mu d \xi d \xi^{\prime}
$$

where $a$ is a smooth classical symbol of order $s \in \mathbb{R}$ in the sense that it satisfies for all multi-indices $\alpha, \alpha^{\prime}, \beta$

$$
\left|\partial_{m}^{\alpha} \partial_{m^{\prime}}^{\alpha^{\prime}} \partial_{\zeta}^{\beta} a\left(m, m^{\prime} ; \zeta\right)\right| \leq C_{\alpha, \alpha^{\prime}, \beta}\left(1+|\zeta|^{2}\right)^{s-|\beta|}
$$

where $m=(x, y) \in \mathbb{R}^{+} \times \mathbb{R}^{n}$ and $\zeta:=\left(\xi, \xi^{\prime}, \mu\right) \in \mathbb{R} \times \mathbb{R} \times \mathbb{R}^{n}$. The integral in (24) makes sense as an oscillatory integral: we integrate by parts a sufficient number $N$ of times in $\zeta$ to get $\Delta_{\zeta}^{N} a\left(m, m^{\prime} ; \zeta\right)$ uniformly $L^{1}$ in $\zeta$; of course we pick up a singularity of the form $\left(x^{2}+x^{\prime 2}+\left|y-y^{\prime}\right|^{2}\right)^{-N}$ by this process but the outcome still makes sense as an element in the dual of $\dot{C}^{\infty}(\bar{X} \times \bar{X})$. If $\widetilde{X}$ is an open manifold extending $\bar{X}$, such a kernel can be extended to a kernel $\widetilde{K}$ on the manifold $\widetilde{X} \times \widetilde{X}$ so that $\widetilde{K}$ is classically conormal to the embedded closed submanifold $\Delta_{\partial}$. Therefore our kernels (which are extendible distributions on $\bar{X} \times \bar{X}$ ) can freely be considered as restriction of distributional kernels acting on a subset of functions of $\widetilde{X} \times \widetilde{X}$, i.e. the set $\dot{C}^{\infty}(\bar{X} \times \bar{X})$ which corresponds to smooth functions with compact support included in $\bar{X} \times \bar{X}$. Standard arguments of pseudodifferential operator theory show that we can require that $K_{s}$ in charts is, up to a smooth kernel, of the form

$$
K_{s}\left(x, y, x^{\prime}, y^{\prime}\right)=\frac{1}{(2 \pi)^{n+2}} \int_{\mathbb{R}} \int_{\mathbb{R}} \int_{\mathbb{R}^{n}} e^{-i x \xi-i x^{\prime} \xi^{\prime}-i\left(y-y^{\prime}\right) \mu} a\left(y ; \xi, \xi^{\prime}, \mu\right) d \mu d \xi d \xi^{\prime} .
$$

Indeed, it suffices to apply a Taylor expansion of $a\left(x, y, x^{\prime}, y^{\prime} ; \zeta\right)$ at $\Delta_{\partial}=\left\{x=x^{\prime}=y-y^{\prime}=0\right\}$ and use integration by parts to show that the difference obtained by quantizing these symbols and the symbols of the form $a(y, \zeta)$ is given by smooth kernels.

We say that the symbol $a$ is classical of order $s$ if it has an asymptotic expansion as $\zeta:=\left(\xi, \xi^{\prime}, \mu\right) \rightarrow \infty$

$$
a(y ; \zeta) \sim \sum_{j=0}^{\infty} a_{s-j}(y ; \zeta)
$$

where $a_{j}$ are homogeneous functions of degree $s-j$ in $\zeta$. It is clear from their definition that operators in $I^{s}\left(\bar{X} \times \bar{X}, \Delta_{\partial}\right)$ have smooth kernels on $(\bar{X} \times \bar{X}) \backslash \Delta_{\partial}$. Let us consider the diagonal singularity of $K$ when its symbol is classical. 
Lemma 26. An operator $K_{s} \in I^{s-n-2}\left(\bar{X} \times \bar{X}, \Delta_{\partial}\right)$ has a kernel which is the sum of a smooth kernel together with a kernel which is smooth outside $\Delta_{\partial}$ and has an expansion at $\Delta_{\partial}$ in local coordinates $\left(x, y, x^{\prime}, y^{\prime}\right)$ of the form

$$
K_{s}\left(x, y, x^{\prime}, y^{\prime}\right) \sim \begin{cases}R^{-s} \sum_{j=0}^{\infty} R^{j} K^{j}(y, \omega) & \text { if } s \notin \mathbb{Z}, \\ R^{-s} \sum_{j=0}^{\infty} R^{j} K^{j}(y, \omega)+\log (R) \sum_{j=0}^{\infty} R^{j} K^{j, 1}(y, \omega) & \text { if } s \in \mathbb{N}_{0}, \\ R^{-s}\left(\sum_{j=0}^{\infty} R^{j} K^{j}(y, \omega)+\log (R) \sum_{j=0}^{\infty} R^{j} K^{j, 1}(y, \omega)\right) & \text { if } s \in-\mathbb{N}\end{cases}
$$

where $R:=\left(x^{2}+x^{\prime 2}+\left|y-y^{\prime}\right|^{2}\right)^{\frac{1}{2}},\left(x, x^{\prime}, y-y^{\prime}\right):=R \omega$ and $K^{j}, K^{j, 1}$ are smooth.

Proof. Assume $K$ has a classical symbol $a$ like in (25). First, we obviously have that for any $N \in \mathbb{N}, K \in C^{N}(\bar{X} \times \bar{X})$ if $s<-N$. Let us write $t=s-n-2$, then we remark that for all $y$, the homogeneous function $a_{t-j}(y,$.$) has a unique homogeneous extension as a homogeneous$ distribution on $\mathbb{R}^{n+2}$ of order $t-j$ if $s \notin j-\mathbb{N}_{0}$ (see [26, Th 3.2.3]), and its Fourier transform is homogeneous of order $-s+j$. Clearly, $K\left(x, y, x^{\prime}, y^{\prime}\right)$ can be written as the Fourier transform in the distribution sense in $\zeta$ of $A_{N}+B_{N}$ where for $N \in \mathbb{N}$

$$
A_{N}(y, \zeta):=\sum_{j=0}^{N} a_{t-j}(y ; \zeta), \quad B_{N}(y, \zeta):=a(y, \zeta)-A_{N}(y, \zeta) .
$$

Now $|\zeta|^{-s+N} B_{N}(y, \zeta)$ is in $L^{1}(d \zeta)$ in $|\zeta|>1$ thus $\mathcal{F}_{\zeta \rightarrow Z}\left((1-\chi(\zeta)) B_{N}(y, \zeta)\right)$ is in $C^{[N-s]}$ with respect to all variables if $\chi \in C_{0}^{\infty}\left(\mathbb{R}^{n+2}\right)$ equals 1 near 0 , while the Fourier transform $\mathcal{F}\left(\chi B_{N}\right)$ and $\mathcal{F}\left(\chi A_{N}\right)$ have the same regularity and are smooth since the convolution of $\mathcal{F}(\chi)$ with a homogeneous function is smooth. This implies the expansion of $K$ at the diagonal when $t \notin \mathbb{Z}$.

For the case $t \in \mathbb{Z}$, this is similar but a bit more complicated. We shall be brief and refer to Beals-Greiner [3, Chap 3.15] for more details (this is done for the Heisenberg calculus there but their proof obviously contains the classical case). Let us denote $\delta_{\lambda}$ the action of dilation by $\lambda \in \mathbb{R}^{+}$on the space $\mathcal{S}^{\prime}$ of tempered distributions on $\mathbb{R}^{n+2}$, then any homogeneous function $f_{k}$ of degree $-n-2-k \in-n-2-\mathbb{N}_{0}$ on $\mathbb{R}^{n+2}$ can be extended to a distribution $\widetilde{f_{k}} \in \mathcal{S}^{\prime}$ satisfying

$$
\delta_{\lambda}\left(\widetilde{f}_{k}\right)=\lambda^{-n-2-k} \widetilde{f}_{k}+\lambda^{-n-2-k} \log (\lambda) P_{k}
$$

for some $P_{k} \in \mathcal{S}^{\prime}$ of order $k$ supported at 0 . This element $P_{k}$ is zero if and only if $f_{k}$ can be extended as a homogeneous distribution on $\mathbb{R}^{n+2}$, or equivalently

$$
\int_{S^{n+1}} f_{k}(\omega) \omega^{\alpha} d \omega=0, \quad \forall \alpha \in \mathbb{N}_{0}^{n+2} \text { with }|\alpha|=k .
$$

According to Proposition 15.30 of [3], the distribution $\widetilde{f}_{k}$ has its Fourier transform which can be written outside 0 as

$$
\mathcal{F}\left(\widetilde{f}_{k}\right)(Z)=L_{k}(Z)+M_{k}(Z) \log |Z|
$$

where $L_{k}$ is a homogeneous function of degree $k$ on $\mathbb{R}^{n+2} \backslash\{0\}$ and $M_{k}$ a homogeneous polynomial of degree $k$. Thus reasoning as above when $t \notin \mathbb{Z}$, this concludes the proof. It can be noted from (28) that in the expansion at $\Delta_{\partial}$ in (26), one has $K^{j, 1}=0$ for all $j=0, \ldots, k$ for some $k \in \mathbb{N}$ if the symbols satisfy the condition

$$
\int_{S^{n+1}} a_{-n-2-j}(y, \omega) \omega^{\alpha} d \omega=0, \quad \forall \alpha \in \mathbb{N}_{0}^{n+2} \text { with }|\alpha|=j
$$


for all $j=0, \ldots, k$ and all $y \in M$. Using the expression of the symbol expansion after a change of coordinates, it is straightforward to check that this condition is invariant with respect to the choice of coordinates.

A consequence of this Lemma (or another way to state it) is that if $K \in I^{s-n-2}\left(\bar{X} \times \bar{X}, \Delta_{\partial}\right)$ is classical, then its kernel lifts to a conormal polyhomogeneous distribution on the manifold with corners $\bar{X} \times_{0} \bar{X}$ obtained by blowing-up $\Delta_{\partial}$ inside $\bar{X} \times \bar{X}$ and

$$
\beta^{*} K \in C^{\infty}\left(\bar{X} \times_{0} \bar{X}\right)+ \begin{cases}\rho_{\mathrm{ff}}^{-s} C^{\infty}\left(\bar{X} \times_{0} \bar{X}\right) & \text { if } s \notin \mathbb{Z}, \\ \rho_{\mathrm{ff}}^{-s} C^{\infty}\left(\bar{X} \times_{0} \bar{X}\right)+\log \left(\rho_{\mathrm{ff}}\right) C^{\infty}\left(\bar{X} \times_{0} \bar{X}\right) & \text { if } s \in \mathbb{N}_{0}, \\ \rho_{\mathrm{ff}}^{-s}\left(C^{\infty}\left(\bar{X} \times_{0} \bar{X}\right)+\log \left(\rho_{\mathrm{ff}}\right) C^{\infty}\left(\bar{X} \times_{0} \bar{X}\right)\right) & \text { if } s \in-\mathbb{N}\end{cases}
$$

Therefore $I^{s}\left(\bar{X} \times \bar{X}, \Delta_{\partial}\right)$ is a subclass of the full 0-calculus of Mazzeo-Melrose [34], in particular with no interior diagonal singularity. Let us make this more precise:

Lemma 27. Let $\ell \in-\mathbb{N}$, then a classical operator $K \in I^{\ell}\left(\bar{X} \times \bar{X}, \Delta_{\partial}\right)$ with a local symbol expansion (25) has a kernel which lifts to $\beta^{*} K \in \rho_{\mathrm{ff}}^{-\ell-n-2} C^{\infty}\left(\bar{X} \times_{0} \bar{X}\right)+C^{\infty}\left(\bar{X} \times_{0} \bar{X}\right)$ if the symbol satisfies the condition (29) for all $j \in \mathbb{N}_{0}$. Conversely, if $K \in C^{-\infty}(\bar{X} \times \bar{X})$ is a distribution which lifts to $\beta^{*} K$ in $\rho_{\mathrm{ff}}^{-\ell-n-2} C^{\infty}\left(\bar{X} \times_{0} \bar{X}\right)+C^{\infty}\left(\bar{X} \times_{0} \bar{X}\right)$, then it is the kernel of a classical operator in $I^{-n-2}\left(\bar{X} \times \bar{X}, \Delta_{\partial}\right)$ with a symbol satisfying (29) for all $j \in \mathbb{N}_{0}$.

Proof. Let us start with the converse: we can extend smoothly the kernel $\beta^{*} K$ to the blownup space $\left[\widetilde{X} \times \widetilde{X}, \Delta_{\partial}\right]$ where $\widetilde{X}$ is an open manifold extending smoothly $\bar{X}$. Then the extended function has an expansion to all order in polar coordinates $(R, \omega)$ at $\{R=0\}$ (i.e. around $\left.\Delta_{\partial}\right)$ where $R=\left(x^{2}+x^{\prime 2}+\left|y-y^{\prime}\right|^{2}\right)^{\frac{1}{2}}$ and $R \omega=\left(x, x^{\prime}, y-y^{\prime}\right)$

$$
K\left(x, y, x^{\prime}, y^{\prime}\right)-\sum_{j=0}^{k} R^{-\ell-n-2+j} K^{j}(y, \omega) \in C^{k}(\bar{X} \times \bar{X}), \quad \forall k \in \mathbb{N}
$$

for some smooth $K^{j}$, in particular using Fourier transform in $Z=\left(x, x^{\prime}, y-y^{\prime}\right)$ one finds that for all $k \in \mathbb{N}$, there exists a classical symbol $a^{k}(y, \zeta)$

$$
K\left(x, y, x^{\prime}, y^{\prime}\right)-\frac{1}{(2 \pi)^{n+2}} \int e^{i x \xi+i x^{\prime} \xi^{\prime}+i\left(y-y^{\prime}\right) \mu} a^{k}\left(y ; \xi, \xi^{\prime}, \mu\right) d \xi d \xi^{\prime} d \mu \in C^{k}(\bar{X} \times \bar{X})
$$

with $a^{k}$ being equal to $\sum_{j=0}^{k} a_{j}^{k}(y ; \zeta)$ when $|\zeta|>1$ for some homogeneous functions $a_{j}^{k}$ of degree $\ell-j$. Moreover, the $a_{j}^{k}$ can be extended as homogeneous distribution on $\mathbb{R}^{n+2}$ since they are given by Fourier transforms of the homogeneous distributions $K^{j}(y, Z)$ in the variable $Z$. Using that a homogeneous function on $\mathbb{R}^{n+2} \backslash\{0\}$ which extends as a homogeneous distribution on $\mathbb{R}^{n+2}$ has no $\log \lambda$ terms in (27), or equivalently satisfies (28), this ends one way.

To prove the first statement, it suffices to consider the kernel in local coordinates and locally $\beta^{*} K$ has the structure (30) with no $\log \left(\rho_{\mathrm{ff}}\right)$ if the local symbol satisfies (29). Notice that having locally the structure $\rho_{\mathrm{ff}}^{-s} C^{\infty}(\bar{X} \times \bar{X})$ for a function is a property which is independent of the choice of coordinates. But from what we just proved above, this implies that in any choice of coordinates the local symbol satisfies (29).

We shall call the subclass of operators in Lemma 27 the class of log-free classical operators of order $\ell \in-\mathbb{N}$, and denote it $I_{\mathrm{lf}}^{\ell}\left(\bar{X} \times \bar{X}, \Delta_{\partial}\right)$.

For (log-free if $s \in-\mathbb{N}$ ) classical operators in $I^{s}\left(\bar{X} \times \bar{X}, \Delta_{\partial}\right)$, there is also a notion of principal symbol which is defined as a homogeneous section of degree $s$ of the conormal bundle 
$N^{*} \Delta_{\partial}$ : if $a$ has an expansion $a(y, \zeta) \sim \sum_{j=0}^{\infty} a_{s-j}(y, \zeta)$ as $\zeta \rightarrow \infty$ with $a_{s-j}$ homogeneous of degree $s-j$ in $\zeta$, then the principal symbol is given by $\sigma_{\mathrm{pr}}(K)=a_{s}$. The principal symbol is actually not invariantly defined if one considers $K$ as an extendible distribution on $\bar{X} \times \bar{X}$ : if $a(y, \zeta)$ and $a^{\prime}(y, \zeta)$ are two classical symbols for the kernel $K$, then if $Z=\left(x, x^{\prime}, z\right)$

$$
\mathcal{F}_{\zeta \rightarrow Z}\left(a_{s}(y, \zeta)-a_{s}^{\prime}(y, \zeta)\right)=0 \text { when } x>0 \text { and } x^{\prime}>0,
$$

thus it is defined only up to this equivalence relation.

To make the correspondence with the 0-calculus of Mazzeo-Melrose [34], we recall that the normal operator of an operator $K \in C^{\infty}\left(\bar{X} \times_{0} \bar{X}\right)$ is given by the restriction to the front face: if $y \in \Delta_{\partial}, N_{y}(K):=\left.K\right|_{\mathrm{ff}_{y}}$ where $\mathrm{ff}_{y}$ is the fiber at $y$ of the unit interior pointing spherical normal bundle $S^{+} N \Delta_{\partial}$ of $\Delta_{\partial}$ inside $\bar{X} \times \bar{X}$, then we remark that the normal operator at $y \in \Delta_{\partial}$ of an admissible operator $K \in I_{\mathrm{lf}}^{-n-2}\left(\bar{X} \times \bar{X} ; \Delta_{\partial}\right)$ is given by the homogeneous function of degree 0 on $\mathbb{R}^{+} \times \mathbb{R}^{+} \times \mathbb{R}^{n} \simeq \mathrm{ff}_{y} \times \mathbb{R}_{+}$

$$
N_{y}(K)(Z)=\mathcal{F}_{\zeta \rightarrow Z}\left(\sigma_{\mathrm{pr}}(K)(y, \zeta)\right) .
$$

B.2. Operators from $\bar{X}$ to $\partial \bar{X}$ and conversely. We define operators in $I^{s}\left(\bar{X} \times \partial \bar{X}, \Delta_{\partial}\right)$ and $I^{s}\left(\partial \bar{X} \times \bar{X}, \Delta_{\partial}\right)$ by saying that their respective distributional kernels are the sum of a smooth kernel on $\bar{X} \times \partial \bar{X}$ (resp. $\partial \bar{X} \times \bar{X}$ ) and of a singular kernel $K_{s} \in C^{-\infty}(\bar{X} \times \partial \bar{X})$ (resp. $\left.L_{s} \in C^{-\infty}(\partial \bar{X} \times \bar{X})\right)$ supported near $\Delta_{\partial}$ of the form (in local coordinates)

$$
\begin{aligned}
& K_{s}\left(x, y, y^{\prime}\right)=\frac{1}{(2 \pi)^{n+1}} \int e^{-i x \xi+i\left(y-y^{\prime}\right) \mu} a\left(y^{\prime} ; \xi, \mu\right) d \xi d \mu, \\
& L_{s}\left(y, x^{\prime}, y^{\prime}\right)=\frac{1}{(2 \pi)^{n+1}} \int e^{i x^{\prime} \xi^{\prime}+i\left(y-y^{\prime}\right) \mu} b\left(y ; \xi^{\prime}, \mu\right) d \xi^{\prime} d \mu
\end{aligned}
$$

with $a$ and $b$ some smooth symbols

$$
\left|\partial_{y}^{\alpha} \partial_{\zeta}^{\beta} a(y, \zeta)\right| \leq C_{\alpha, \beta}\langle\zeta\rangle^{s-|\beta|}, \quad \quad\left|\partial_{y}^{\alpha} \partial_{\zeta}^{\beta} b(y, \zeta)\right| \leq C_{\alpha, \beta}\langle\zeta\rangle^{s-|\beta|}
$$

for all $\alpha, \beta$. We shall say they are classical if their symbols have an expansion in homogeneous functions at $\zeta \rightarrow \infty$, just like above for operators on $\bar{X}$. It is easy to see that such operators map respectively $\dot{C}^{\infty}(\bar{X})$ to $C^{\infty}(\partial \bar{X})$ and $C^{\infty}(\partial \bar{X})$ to $C^{-\infty}(\bar{X}) \cap C^{\infty}(X)$.

Using the exact same arguments as for operators on $\bar{X}$, we have the following

Lemma 28. Let $\ell \in-\mathbb{N}$, then a classical operator $K \in I^{\ell}\left(\bar{X} \times \partial \bar{X}, \Delta_{\partial}\right)$ with a local symbol expansion $a(y, \zeta) \sim \sum_{j=0}^{\infty} a_{-n-1-j}(y, \zeta)$ has a kernel which lifts to $\beta_{1}^{*} K \in \rho_{\mathrm{ff}}^{-\ell-n-1} C^{\infty}\left(\bar{X} \times_{0}\right.$ $\partial \bar{X})+C^{\infty}\left(\bar{X} \times_{0} \partial \bar{X}\right)$ if

$$
\int_{S^{n}} a_{-n-1-j}(y, \omega) \omega^{\alpha} d \omega=0, \quad \forall \alpha \in \mathbb{N}_{0}^{n+1} \text { with }|\alpha|=j
$$

for all $j \in \mathbb{N}_{0}$. Conversely, if $K \in C^{-\infty}(\bar{X} \times \bar{\partial} X)$ is a distribution which lifts to $\beta_{1}^{*} K$ in $\rho_{\mathrm{ff}}^{-\ell-n-1} C^{\infty}\left(\bar{X} \times_{0} \partial \bar{X}\right)+C^{\infty}\left(\bar{X} \times_{0} \partial \bar{X}\right)$, then it is the kernel of a classical operator in $I^{\ell}\left(\bar{X} \times \partial \bar{X}, \Delta_{\partial}\right)$ with a symbol satisfying $(29)$ for all $j \in \mathbb{N}_{0}$. The symmetric statement holds for operators in $I^{\ell}\left(\partial \bar{X} \times \bar{X}, \Delta_{\partial}\right)$.

We shall also call the operators of Lemma 28 log-free classical operators and denote this class by $I_{\mathrm{lf}}^{\ell}\left(\bar{X} \times \partial \bar{X}, \Delta_{\partial}\right)$ and $I_{\mathrm{lf}}^{\ell}\left(\partial \bar{X} \times \bar{X}, \Delta_{\partial}\right)$.

Notice that, since the restriction of a function in $C^{\infty}\left(\bar{X} \times_{0} \bar{X}\right)$ to the right boundary gives a function in $C^{\infty}\left(\bar{X} \times_{0} \partial \bar{X}\right)$, we deduce that an operator $I^{-n-2}\left(\bar{X} \times \bar{X}, \Delta_{\partial}\right)$ satisfying condition (29) induces naturally (by restriction to the boundary on the right variable) an operator in 
$I^{-n-1}\left(\bar{X} \times \partial \bar{X}, \Delta_{\partial}\right)$ satisfying (32). This can also be seen by considering the oscillatory integrals restricted to $x^{\prime}=0$ but it is more complicated to prove.

B.3. Compositions. We start with a result on the composition of operators mapping from $\bar{X}$ to $M$ with operators mapping $M$ to $M$ or $M$ to $\bar{X}$. This is will be done using the pushforward theorem of Melrose [35, Th. 5]

Proposition 29. Let $A: C^{\infty}\left(M ;{ }^{0} \Sigma \otimes \Omega^{\frac{1}{2}}\right) \rightarrow C^{\infty}\left(M ;{ }^{0} \Sigma \otimes \Omega^{\frac{1}{2}}\right)$ be a pseudo-differential operator of negative order with lifted kernel in $\mathcal{A}_{\mathrm{phg}}^{E_{\mathrm{ff}}}\left(M \times_{0} M ; \mathcal{E} \otimes \Omega_{b}^{\frac{1}{2}}\right)$. Let $B: \dot{C}^{\infty}\left(\bar{X} ;{ }^{0} \Sigma \otimes\right.$ $\left.\Omega_{b}^{\frac{1}{2}}\right) \rightarrow C^{\infty}\left(M ;{ }^{0} \Sigma \otimes \Omega^{\frac{1}{2}}\right)$ be an operator with lifted kernel in $\mathcal{A}_{\mathrm{ph}}^{F_{\mathrm{ff}}, F_{\mathrm{rb}}}\left(M \times_{0} \bar{X} ; \mathcal{E}_{r} \otimes \Omega_{b}^{\frac{1}{2}}\right)$ and let $C: C^{\infty}\left(M ;{ }^{0} \Sigma \otimes \Omega^{\frac{1}{2}}\right) \rightarrow C^{-\infty}\left(\bar{X} ;{ }^{0} \Sigma \otimes \Omega_{b}^{\frac{1}{2}}\right)$ be an operator with lifted kernel on $\mathcal{A}_{\mathrm{phg}}^{G_{\mathrm{ff}}, G_{\mathrm{lb}}}\left(\bar{X} \times_{0}\right.$ $\left.M ; \varepsilon_{l} \otimes \Omega_{b}^{\frac{1}{2}}\right)$. Then the Schwartz kernels of $A \circ B$ and $C \circ B$ lift to polyhomogeneous conormal kernels

and the index sets satisfy

$$
\begin{aligned}
& k_{A \circ B} \in \mathcal{A}_{\mathrm{phg}}^{H_{\mathrm{ff}}, H_{\mathrm{rb}}}\left(M \times_{0} \bar{X} ; \mathcal{E}_{r} \otimes \Omega_{b}^{\frac{1}{2}}\right) \\
& k_{C \circ B} \in \mathcal{A}_{\mathrm{phg}}^{I_{\mathrm{ff}}, I_{\mathrm{lb}}, I_{\mathrm{rb}}}\left(\bar{X} \times_{0} \bar{X} ; \mathcal{E} \otimes \Omega_{b}^{\frac{1}{2}}\right)
\end{aligned}
$$

$$
\begin{aligned}
& H_{\mathrm{ff}}=\left(E_{\mathrm{ff}}+F_{\mathrm{ff}}+\frac{n}{2}\right) \varpi\left(F_{\mathrm{rb}}+\frac{n}{2}\right), \quad H_{\mathrm{rb}}=F_{\mathrm{rb}} \bar{U}\left(F_{\mathrm{ff}}+\frac{n}{2}\right) \\
& I_{\mathrm{ff}}=\left(F_{\mathrm{ff}}+G_{\mathrm{ff}}+\frac{n}{2}\right) \cup\left(F_{\mathrm{rb}}+G_{\mathrm{lb}}+\frac{n}{2}\right), \quad I_{\mathrm{lb}}=G_{\mathrm{lb}} \cup\left(G_{\mathrm{ff}}+\frac{n}{2}\right), \quad I_{\mathrm{rb}}=F_{\mathrm{rb}} \bar{U}\left(F_{\mathrm{ff}}+\frac{n}{2}\right) .
\end{aligned}
$$

Proof. The proof is an application of Melrose push-forward theorem. Let us discuss first the composition $A \circ B$. We denote by $\Delta$ both the diagonal in $M \times M$ and the submanifold $\left\{\left(m, m^{\prime}\right) \in M \times \bar{X} ; m=m^{\prime}\right\}$, by $\left(\pi_{j}\right)_{j=l, c, r}$ the canonical projections of $M \times M \times \bar{X}$ obtained by projecting-off the $j$ factor (here $l, c, r$ mean left, center, right), and let

$$
\Delta_{3}:=\left\{\left(m, m^{\prime}, m^{\prime \prime}\right) \in M \times M \times \bar{X} ; m=m^{\prime}=m^{\prime \prime}\right\}, \quad \Delta_{2, j}=\pi_{j}^{-1}(\Delta) \text { for } j=l, c, r .
$$

The triple space $M \times_{0} M \times_{0} \bar{X}$ is the iterated blow-up

$$
M \times_{0} M \times_{0} \bar{X}:=\left[M \times M \times \bar{X} ; \Delta_{3}, \Delta_{2, l}, \Delta_{2, c}, \Delta_{2, r}\right] .
$$

The submanifolds to blow-up are $p$-submanifolds, moreover $\Delta_{3}$ is contained in each $\Delta_{2, j}$ and the lifts of $\Delta_{2, j}$ to the blow-up $\left[M \times M \times \bar{X} ; \Delta_{3}\right]$ are disjoint. Consequently (see for instance $\left[23\right.$, Lemma 6.2]) the order of blow-ups can be commuted and the canonical projections $\pi_{j}$ lift to maps

$\beta_{l}: M \times_{0} M \times_{0} \bar{X} \rightarrow M \times_{0} \bar{X}, \beta_{c}: M \times_{0} M \times_{0} \bar{X} \rightarrow M \times_{0} \bar{X}, \beta_{r}: M \times_{0} M \times_{0} \bar{X} \rightarrow M \times_{0} M$ which are $b$-fibrations. The manifold $M \times_{0} M \times_{0} \bar{X}$ has 5 boundary hypersurfaces, the front face $\mathrm{ff}^{\prime}$ obtained by blowing up $\Delta_{3}$, the faces lf, cf, rf obtained from the respective blow-up of $\Delta_{2, l}, \Delta_{2, c}, \Delta_{2, r}$ and finally the face $\mathrm{rb}^{\prime}$ obtained from the lift of the original face $M \times M \times M \subset M \times M \times \bar{X}$. We denote by $\rho_{f}$ a smooth boundary defining function of the face $f \in\left\{\mathrm{ff}^{\prime}, \mathrm{rf}, \mathrm{cf}, \mathrm{lf}, \mathrm{rb}^{\prime}\right\}$. If $k_{A}$ and $k_{B}$ are the lifted kernel of $A$ and $B$ to respectively $M \times_{0} M$ and $M \times_{0} \bar{X}$ then it is possible to write the composition as a push-forward

$$
k_{A \circ B} \cdot \mu=\beta_{c *}\left(\beta_{r}^{*} k_{A} \cdot \beta_{l}^{*} k_{B} \cdot \beta_{c}^{*} \mu\right)
$$


if $\mu \in C^{\infty}\left(M \times_{0} \bar{X} ; \varepsilon_{r} \otimes \Omega_{b}^{\frac{1}{2}}\right)$. An easy computation shows that a smooth b-density $\omega$ on $M \times M \times \bar{X}$ lifts through $\beta$ to an element

$$
\beta^{*} \omega \in \rho_{\mathrm{ff}}^{2 n}\left(\rho_{\mathrm{lf}} \rho_{\mathrm{rf}} \rho_{\mathrm{cf}}\right)^{n} C^{\infty}\left(M \times_{0} M \times_{0} \bar{X} ; \Omega_{b}\right)
$$

so by considering the lifts through $\beta_{l}, \beta_{c}, \beta_{r}$ of boundary defining functions in $M \times_{0} \bar{X}, M \times_{0} \bar{X}$ and $\bar{X} \times_{0} \bar{X}$ respectively we deduce that there is some index set $K=\left(K_{\mathrm{ff}^{\prime}}, K_{\mathrm{rb}^{\prime}}, K_{\mathrm{lf}}, K_{\mathrm{rf}}, K_{\mathrm{cf}}\right)$ such that

$$
\begin{gathered}
\beta_{r}^{*} k_{A} \cdot \beta_{l}^{*} k_{B} \cdot \beta_{c}^{*} \mu \in \mathcal{A}_{\mathrm{phg}}^{K}\left(M \times_{0} M \times_{0} \bar{X} ; \Omega_{b}\right) \\
K_{\mathrm{ff}}=E_{\mathrm{ff}}+F_{\mathrm{ff}}+\frac{n}{2}, \quad K_{\mathrm{rb}^{\prime}}=F_{\mathrm{rb}}, \quad K_{\mathrm{lf}}=F_{\mathrm{ff}}+\frac{n}{2} \\
K_{\mathrm{rf}}=E_{\mathrm{ff}}+\frac{n}{2}, \quad K_{\mathrm{cf}}=F_{\mathrm{rb}}+\frac{n}{2} .
\end{gathered}
$$

Then from the push-forward theorem of Melrose [35, Th. 5], we obtain that

$$
\begin{gathered}
\left(\beta_{c}\right)_{*}\left(\beta_{r}^{*} k_{A} \cdot \beta_{l}^{*} k_{B} \cdot \beta_{c}^{*} \mu\right) \in \mathcal{A}_{\mathrm{phg}}^{H_{\mathrm{ff}}, H_{\mathrm{rb}}}\left(M \times_{0} \bar{X}, \Omega_{b}\right) \\
H_{\mathrm{ff}}=\left(E_{\mathrm{ff}}+F_{\mathrm{ff}}+\frac{n}{2}\right) \bar{\cup}\left(F_{\mathrm{rb}}+\frac{n}{2}\right), \quad H_{\mathrm{rb}}=F_{\mathrm{rb}} \bar{\cup}\left(F_{\mathrm{ff}}+\frac{n}{2}\right)
\end{gathered}
$$

and this shows the first composition result for $A \circ B$. Remark that to apply [35, Th.5], we need the index of $K_{\mathrm{rf}}>0$, i.e. $E_{\mathrm{ff}}+n / 2>0$, but this is automatically satisfied with our assumption that $A$ is a pseudodifferential operator of negative order on $M$.

The second composition result is very similar, except that there are more boundary faces to consider. One defines $\Delta_{3}:=\left\{\left(m, m^{\prime}, m^{\prime \prime}\right) \in \bar{X} \times M \times \bar{X} ; m=m^{\prime}=m^{\prime \prime}\right\}$ and let

$$
\Delta_{2, j}=\left\{\left(m_{l}, m_{c}, m_{r}\right) \in \bar{X} \times M \times \bar{X} ; m_{i}=m_{k} \text { if } j \notin\{i, k\}\right\}
$$

similarly as before. The triple space is defined like (33), it has now 6 boundary faces which we denote as in the case above but with the additional face, denoted $\mathrm{lb}^{\prime}$, obtained from the lift of the original boundary $M \times M \times \bar{X}$. The same arguments as above show that the canonical projections from $\bar{X} \times_{0} M \times_{0} \bar{X}$ obtained by projecting-off one factor lift to b-fibrations $\beta_{r}, \beta_{l}, \beta_{c}$ from the triple space to $\bar{X} \times_{0} M, M \times_{0} \bar{X}$ and $\bar{X} \times_{0} \bar{X}$. Like for the case above, one has to push-forward a distribution $\beta_{r}^{*} k_{C} \cdot \beta_{l}^{*} k_{B} \cdot \beta_{c}^{*} \mu$, and a computation gives that there is an index set $L=\left(L_{\mathrm{ff}^{\prime}}, L_{\mathrm{rb}^{\prime}}, L_{\mathrm{lb}^{\prime}}, L_{\mathrm{lf}}, L_{\mathrm{rf}}, L_{\mathrm{cf}}\right)$ such that

$$
\begin{gathered}
\beta_{r}^{*} k_{C} \cdot \beta_{l}^{*} k_{B} \cdot \beta_{c}^{*} \mu \in \mathcal{A}_{\mathrm{phg}}^{L}\left(\bar{X} \times_{0} M \times_{0} \bar{X} ; \Omega_{b}\right) \\
L_{\mathrm{ff}}=F_{\mathrm{ff}}+G_{\mathrm{ff}}+\frac{n}{2}, \quad L_{\mathrm{rb}^{\prime}}=F_{\mathrm{rb}}, \quad L_{\mathrm{lb}^{\prime}}=G_{\mathrm{lb}}, \\
L_{\mathrm{lf}}=F_{\mathrm{ff}}+\frac{n}{2}, \quad L_{\mathrm{rf}}=G_{\mathrm{ff}}+\frac{n}{2}, \quad L_{\mathrm{cf}}=F_{\mathrm{rb}}+G_{\mathrm{rb}}+\frac{n}{2}
\end{gathered}
$$

and by pushing forward through $\beta_{c}$ using Melrose [35, Th. 5], we deduce that the result is polyhomogeneous conormal on $\bar{X} \times_{0} \bar{X}$ with the desired index set.

In order to analyze the composition $K^{*} K$ in Subsection 3.2, we use the symbolic approach since it is a slightly more precise (in terms of log terms at the diagonal) than the push-forward Theorem in this case, and a bit easier to compute the principal symbol of the composition. We are led to study the composition between log-free classical operators $K$ and $L$ where $K: C^{\infty}(\bar{X}) \rightarrow C^{\infty}(\partial \bar{X})$ is an operator in $I_{\mathrm{lf}}^{-1}(\bar{X} \times \partial \bar{X})$ and $L: C^{\infty}(\partial \bar{X}) \rightarrow C^{\infty}(\bar{X})$ is in $I_{\mathrm{lf}}^{-1}(\partial \bar{X} \times \bar{X})$. We show 
Lemma 30. Let $K \in I_{\mathrm{lf}}^{-1}(\bar{X} \times \partial \bar{X})$ and $L \in I_{\mathrm{lf}}^{-1}(\partial \bar{X} \times \bar{X})$ with principal symbols $\sigma_{K}(y ; \xi, \mu)$ and $\sigma_{L}(y ; \xi, \mu)$. The composition $L \circ K$ is a classical pseudodifferential operator on $\partial \bar{X}$ in the class $L \circ K \in \Psi^{-1}(\partial \bar{X})$. Moreover the principal symbol of $L K$ is given by

$$
\sigma_{\operatorname{pr}}(L \circ K)(y ; \mu)=(2 \pi)^{-2} \int_{0}^{\infty} \hat{\sigma}_{L}(y ;-x, \mu) . \hat{\sigma}_{K}(y ; x, \mu) d x .
$$

where $\hat{\sigma}$ denotes the Fourier transform of $\sigma$ in the variable $\xi$.

Proof. Since the composition with smoothing operators is easier, we essentially need to understand the composition of singular kernels like (31). Writing the kernel of $K$ and $L$ as a sum of elements $K_{j}, L_{j}$ of the form (31), we are reduced to analyze in a chart $U$

$$
L_{j} K_{j} f(y)=\frac{1}{(2 \pi)^{2 n+2}} \int e^{i x^{\prime}\left(\xi^{\prime}-\xi\right)+i y^{\prime}\left(\mu^{\prime}-\mu\right)+i y \mu-i y^{\prime \prime} \mu^{\prime}} b\left(y ; \xi^{\prime}, \mu\right) \chi\left(x^{\prime}, y^{\prime}\right) a\left(y^{\prime \prime} ; \xi, \mu^{\prime}\right) f\left(y^{\prime \prime}\right) d y^{\prime \prime} d \Omega
$$

where $d \Omega:=d y^{\prime} d x^{\prime} d \xi d \xi^{\prime} d \mu d \mu^{\prime}, \chi \in C_{0}^{\infty}(U)$ and $a, b$ are compactly supported in $U$ in the $y$ and $y^{\prime \prime}$ coordinates. If $U$ intersects the boundary $\partial \bar{X}$, then $\chi$ is supported in $x^{\prime} \geq 0$. The kernel of the composition $L_{j} K_{j}$ in the chart $U$ is then

$$
\begin{aligned}
F\left(y, y^{\prime \prime}\right) & =\frac{1}{(2 \pi)^{2 n+2}} \int e^{i x^{\prime}\left(\xi^{\prime}-\xi\right)+i y^{\prime}\left(\mu^{\prime}-\mu\right)+i y \mu-i y^{\prime \prime} \mu^{\prime}} b\left(y ; \xi^{\prime}, \mu\right) \chi\left(x^{\prime}, y^{\prime}\right) a\left(y^{\prime \prime} ; \xi, \mu^{\prime}\right) d \Omega \\
& =\frac{1}{(2 \pi)^{n}} \int e^{i \mu\left(y-y^{\prime \prime}\right)} c\left(y, y^{\prime \prime} ; \mu\right) d \mu
\end{aligned}
$$

where

$$
c\left(y, y^{\prime \prime} ; \mu\right):=\frac{1}{(2 \pi)^{n+2}} \int e^{-i y^{\prime \prime} \cdot \mu^{\prime}} b\left(y ; \xi^{\prime}, \mu\right) a\left(y^{\prime \prime} ; \xi, \mu-\mu^{\prime}\right) \hat{\chi}\left(\xi-\xi^{\prime}, \mu^{\prime}\right) d \mu^{\prime} d \xi d \xi^{\prime} .
$$

We want to prove that $c\left(y, y^{\prime \prime} ; \mu\right)$ is a symbol of order -1 with an expansion in homogeneous terms in $\mu$ as $\mu \rightarrow \infty$. We shall only consider the case where $U \cap \partial \bar{X} \neq \emptyset$ since the other case is simpler. First, remark that in $U$ the function $\chi$ can be taken of the form $\chi(x, y)=\varphi(x) \psi(y)$ with $\psi \in C_{0}^{\infty}\left(\mathbb{R}^{n}\right)$ and $\varphi \in C_{0}^{\infty}([0,1))$ equal to 1 in $[0,1 / 2]$, therefore $\hat{\chi}(\xi, \mu)=\hat{\varphi}(\xi) \hat{\psi}(\mu)$ with $\hat{\psi}$ Schwartz and by integration by parts one also has

$$
\hat{\varphi}(\xi)=\frac{1}{i \xi}\left(1+\hat{\varphi}^{\prime}(\xi)\right) \text {. }
$$

with $\hat{\varphi}^{\prime}$ Schwartz. We first claim that $\left|\partial_{y}^{\alpha} \partial_{y^{\prime \prime}}^{\beta} \partial_{\mu}^{\gamma} c\left(y, y^{\prime \prime} ; \mu\right)\right| \leq C\langle\mu\rangle^{-1-|\gamma|}$ uniformly in $y, y^{\prime \prime}$ : indeed using the properties of $\hat{\chi}$ and the symbolic assumptions on $a, b$, we have that for any $N \gg|\beta|$, there is a constant $C>0$ such that

$$
\begin{gathered}
\left|\partial_{y}^{\alpha} \partial_{y^{\prime \prime}}^{\beta} \partial_{\mu}^{\gamma} c\left(y, y^{\prime \prime} ; \mu\right)\right| \leq \\
C \int\left(\frac{1}{1+|\xi|^{\prime}+|\mu|}\right)^{1+k}\left(\frac{1}{1+|\xi|+|\mu|}\right)^{1+j}\left\langle\xi-\xi^{\prime}\right\rangle^{-1}\left\langle\mu^{\prime}\right\rangle^{-N+|\beta|} d \mu^{\prime} d \xi d \xi^{\prime}
\end{gathered}
$$

where $j+k=|\gamma|$. Using polar coordinates $\left(\xi, \xi^{\prime}\right)=r e^{i \theta}$ in $\mathbb{C} \simeq \mathbb{R}^{2}$, the integral above is bounded by

$$
C \int\left(\frac{1}{1+r|\cos (\theta)|+|\mu|}\right)^{1+k}\left(\frac{1}{1+r|\sin \theta|+|\mu|}\right)^{1+j} \frac{1}{1+r|\cos \theta-\sin \theta|} r d r d \theta
$$

which, by a change of variable $r \rightarrow r|\mu|$ and splitting the $\theta$ integral in different regions, is easily shown to be bounded by $C\langle\mu\rangle^{-1-|\beta|}$. 
To prove that $L K$ it is a classical operator of order -1 (with an expansion in homogeneous terms), we can modify slightly the usual proof of composition of pseudo-differential operators, like in Theorem 3.4 of [21]. Let $\theta \in C_{0}^{\infty}(\mathbb{R})$ be an even function equal to 1 near 0 . We write for $\mu=\lambda \omega$ with $\omega \in S^{n-1}$

$$
\begin{aligned}
F\left(y, y^{\prime \prime}\right) & =\frac{1}{(2 \pi)^{2 n+2}} \int e^{i x^{\prime}\left(\xi^{\prime}-\xi\right)-i\left(\mu^{\prime}-\mu\right)\left(y^{\prime \prime}-y^{\prime}\right)} \chi\left(x^{\prime}, y^{\prime}\right) b\left(y ; \xi^{\prime}, \mu\right) a\left(y^{\prime \prime} ; \xi, \mu^{\prime}\right) d y^{\prime} d x^{\prime} d \xi d \mu d \mu^{\prime} d \xi^{\prime} \\
& =\frac{1}{(2 \pi)^{n}} \int e^{i\left(y-y^{\prime \prime}\right) \mu} c\left(y, y^{\prime \prime} ; \mu\right) d \mu
\end{aligned}
$$

with

$$
\begin{aligned}
& c\left(y, y^{\prime} ; \mu\right) \\
= & \frac{\lambda^{n+1}}{(2 \pi)^{n+2}} \int e^{-i \lambda x^{\prime} \zeta-i \lambda \sigma . s} \chi\left(x^{\prime}, y^{\prime \prime}-s\right) b\left(y ; \xi^{\prime}, \lambda \omega\right) a\left(y^{\prime \prime} ; \xi^{\prime}+\lambda \zeta, \lambda(\omega+\sigma)\right) d \Omega d \xi^{\prime} \\
= & \frac{\lambda^{n+1}}{(2 \pi)^{n+2}} \int e^{-i \lambda x^{\prime} \zeta-i \lambda \sigma . s} \varphi\left(x^{\prime}\right) \theta(\zeta) \psi\left(y^{\prime \prime}-s\right) b\left(y ; \xi^{\prime}, \lambda \omega\right) a\left(y^{\prime \prime} ; \xi^{\prime}+\lambda \zeta, \lambda(\omega+\sigma)\right) d \Omega d \xi^{\prime} \\
& +\frac{\lambda^{n+1}}{(2 \pi)^{n+2}} \int e^{-i \lambda x^{\prime} \zeta-i \lambda \sigma . s} \varphi\left(x^{\prime}\right)(1-\theta)(\zeta) \psi\left(y^{\prime \prime}-s\right) b\left(y ; \xi^{\prime}, \lambda \omega\right) a\left(y^{\prime \prime} ; \xi^{\prime}+\lambda \zeta, \lambda(\omega+\sigma)\right) d \Omega d \xi^{\prime} \\
= & c_{1}\left(y, y^{\prime \prime} ; \mu\right)+c_{2}\left(y, y^{\prime \prime} ; \mu\right)
\end{aligned}
$$

where $\Omega=\left(\sigma, s, \zeta, x^{\prime}\right)$. Let us denote the phase by $\Phi:=x^{\prime} \zeta+\sigma . s$. The last integral can be dealt with by integrating by part in $x^{\prime}$ :

$$
\begin{gathered}
c_{2}\left(y, y^{\prime \prime} ; \mu\right)= \\
\frac{\lambda^{n}}{i(2 \pi)^{n+2}} \int e^{-i \lambda \Phi} \varphi^{\prime}\left(x^{\prime}\right) \frac{(1-\theta)(\zeta)}{\zeta} \psi\left(y^{\prime \prime}-s\right) b\left(y ; \xi^{\prime}, \lambda \omega\right) a\left(y^{\prime \prime} ; \xi^{\prime}+\lambda \zeta, \lambda(\omega+\sigma)\right) d \Omega d \xi^{\prime} \\
+\frac{\lambda^{n}}{i(2 \pi)^{n+2}} \int e^{-i \lambda \sigma \cdot s} \frac{(1-\theta)(\zeta)}{\zeta} \psi\left(y^{\prime \prime}-s\right) b\left(y ; \xi^{\prime}, \lambda \omega\right) a\left(y^{\prime \prime} ; \xi^{\prime}+\lambda \zeta, \lambda(\omega+\sigma)\right) d \sigma d s d \zeta d \xi^{\prime} .
\end{gathered}
$$

We can extend $\varphi^{\prime}=\partial_{x} \varphi$ by 0 on $(-\infty, 0]$ to obtain a $C_{0}^{\infty}(\mathbb{R})$ function which vanishes near 0 . Since $(1-\theta)$ vanishes near 0 , one easily prove that the first integral in (35) is a $O\left(\lambda^{-N}\right)$ for all $N$, uniformly in $y, y^{\prime \prime}$ by using integrations by parts $N$ times in $\zeta$ and $\partial_{\zeta}\left(e^{-i \lambda x^{\prime} \zeta}\right)=$ $-i \lambda \zeta e^{-i \lambda x^{\prime} \zeta}$. Now for the second integral in (35), we use stationary phase in $(\sigma, s)$, one has for any $N \in \mathbb{N}$

$$
\begin{gathered}
\int e^{-i \lambda \sigma \cdot s} \psi\left(y^{\prime \prime}-s\right) \frac{(1-\theta)(\zeta)}{\zeta} b\left(y ; \xi^{\prime}, \lambda \omega\right) a\left(y^{\prime \prime} ; \xi^{\prime}+\lambda \zeta, \lambda(\omega+\sigma)\right) d \sigma d s \\
=(2 \pi)^{n} \frac{(1-\theta)(\zeta)}{\zeta}\left(\sum_{|\alpha| \leq N} \frac{i^{|\alpha|}}{\alpha !} \partial^{\alpha} \psi\left(y^{\prime \prime}\right) b\left(y ; \xi^{\prime}, \mu\right) \partial_{\mu}^{\alpha} a\left(y^{\prime \prime} ; \xi^{\prime}+\lambda \zeta, \mu\right)+S_{N}\left(y, y^{\prime \prime} ; \xi^{\prime}, \zeta, \mu\right)\right)
\end{gathered}
$$

with $\left|S_{N}\left(y, y^{\prime \prime} ; \xi^{\prime}, \zeta, \mu\right)\right| \leq C\left\langle\left(\xi^{\prime}, \mu\right)\right\rangle^{-1}\left\langle\left(\xi^{\prime}+|\mu| \zeta, \mu\right)\right\rangle^{-1-N}$. Now, both $a$ and $b$ can be written under the form $a=a_{N}+a_{h}$ and $b=b_{h}+b_{N}$ where $a_{N}(y ; \xi, \mu), b_{N}(y ; \xi, \mu)$ are bounded in norm by $C\langle(\xi, \mu)\rangle^{-N}$ and $a_{h}(y ; \xi, \mu), b_{h}(y, \xi, \mu)$ are finite sums of homogeneous functions $a_{h}^{-j}, b_{h}^{-j}$ of order $-j$ in $|(\xi, \mu)|>1$ for $j=1, \ldots N-1$. Replacing $a, b$ in (36) by their decomposition $a_{N}+a_{h}$ and $b_{N}+b_{h}$ we get that $c\left(y, y^{\prime \prime}, \mu\right)$ is the sum of a term bounded uniformly by 
$C\langle\mu\rangle^{-N+2}$ and some terms of the form

$$
\lambda \int \frac{1-\theta(\zeta)}{\zeta} b_{h}^{-j}\left(y ; \xi^{\prime}, \mu\right) \partial^{\alpha} \psi\left(y^{\prime \prime}\right) \partial_{\mu}^{\alpha} a_{h}^{-k}\left(y^{\prime \prime} ; \xi^{\prime}+\lambda \zeta, \mu\right) d \zeta d \xi^{\prime}
$$

The integral is well defined and is easily seen (by changing variable $\xi^{\prime} \rightarrow \lambda \xi^{\prime}$ ) to be homogeneous of order $-k-j-|\alpha|+1$ for $\lambda=|\mu|>1$. This shows that $c_{2}\left(y, y^{\prime \prime} ; \mu\right)$ has an expansion in homogeneous terms. It remains to deal with $c_{1}$. We first apply stationary phase in $(\sigma, s)$ variable and we get

$$
\begin{gathered}
c_{1}\left(y, y^{\prime \prime} ; \mu\right)=\frac{\lambda}{(2 \pi)^{2}} \sum_{|\alpha| \leq N} \frac{i^{|\alpha|}}{\alpha !} \partial^{\alpha} \psi\left(y^{\prime \prime}\right) \int e^{-i \lambda x^{\prime} \zeta} b\left(y, \xi^{\prime}, \mu\right) \varphi\left(x^{\prime}\right) \theta(\zeta) \partial_{\mu}^{\alpha} a\left(y^{\prime \prime} ; \xi^{\prime}+\lambda \zeta, \mu\right) d x^{\prime} d \xi^{\prime} d \zeta \\
+\int \varphi\left(x^{\prime}\right) S_{N}^{\prime}\left(y, y^{\prime \prime} ; \xi^{\prime}, \zeta, \mu\right) d \xi^{\prime} d \zeta d x^{\prime}
\end{gathered}
$$

for some $S_{N}^{\prime}$ which will contributes to $O\left(\lambda^{-N-2}\right)$ as above for $c_{2}$. Decomposing $a(y, \xi, \mu)$ and $b(y, \xi, \mu)$ as above in homogeneous terms outside a compact set in $(\xi, \mu)$, it is easy to see that up to a $O\left(\lambda^{-N}\right)$ term, we can reduce the analysis of $c_{1}\left(y, y^{\prime \prime} ; \mu\right)$ to the case where $a, b$ are replaced by terms $a_{h}^{-j}, b_{h}^{-k}$ homogeneous outside compacts of orders $-j,-k$. We then have

$$
\begin{gathered}
\int e^{-i \lambda x^{\prime} \zeta} b_{h}^{-j}\left(y, \xi^{\prime}, \mu\right) \varphi\left(x^{\prime}\right) \theta(\zeta) \partial_{\mu}^{\alpha} a_{h}^{-k}\left(y^{\prime \prime} ; \xi^{\prime}+\lambda \zeta, \mu\right) d x^{\prime} d \xi^{\prime} d \zeta \\
=\lambda^{-j-k-|\alpha|+1} \int e^{-i \lambda x^{\prime} \zeta} b_{h}^{-j}\left(y, \xi^{\prime}, \omega\right) \varphi\left(x^{\prime}\right) \theta(\zeta) \partial_{\mu}^{\alpha} a_{h}^{-k}\left(y^{\prime \prime} ; \xi^{\prime}+\zeta, \omega\right) d x^{\prime} d \xi^{\prime} d \zeta
\end{gathered}
$$

and we write by Taylor expansion at $\zeta=0$

$$
\theta(\zeta) \partial_{\mu}^{\alpha} a_{h}^{-k}\left(y^{\prime \prime} ; \xi^{\prime}+\zeta, \omega\right)=\theta(\zeta) \partial_{\mu}^{\alpha} a_{h}^{-k}\left(y^{\prime \prime} ; \xi^{\prime}, \omega\right)+\zeta \theta(\zeta) a^{\prime}\left(y^{\prime \prime}, \xi^{\prime}, \zeta, \omega\right)
$$

for some $a^{\prime}\left(y^{\prime \prime} ; \xi^{\prime}, \zeta, \mu\right)$ smooth in $y^{\prime \prime}$ and homogeneous of degree $-k-1$ in $|(\xi, \zeta, \mu)|>1$. For the term with $a^{\prime}$, we have by integration by parts in $x^{\prime}$

$$
\begin{gathered}
\int \zeta e^{-i \lambda x^{\prime} \zeta} b_{h}^{-j}\left(y, \xi^{\prime}, \omega\right) \varphi\left(x^{\prime}\right) \theta(\zeta) \partial_{\mu}^{\alpha} a^{\prime}\left(y^{\prime \prime} ; \xi^{\prime}, \zeta, \omega\right) d x^{\prime} d \xi^{\prime} d \zeta \\
=(i \lambda)^{-1} \int e^{-i \lambda x^{\prime} \zeta} \varphi^{\prime}\left(x^{\prime}\right) b_{h}^{-j}\left(y, \xi^{\prime}, \omega\right) \theta(\zeta) \partial_{\mu}^{\alpha} a^{\prime}\left(y^{\prime \prime} ; \xi^{\prime}, \zeta, \omega\right) d x^{\prime} d \xi^{\prime} d \zeta \\
+(i \lambda)^{-1} \int b_{h}^{-j}\left(y, \xi^{\prime}, \omega\right) \theta(\zeta) \partial_{\mu}^{\alpha} a^{\prime}\left(y^{\prime \prime} ; \xi^{\prime}, \zeta, \omega\right) d \xi^{\prime} d \zeta
\end{gathered}
$$

and the first term is $O\left(\lambda^{-\infty}\right)$ by non-stationary phase while the second one is homogeneous of order -1 in $\lambda$ (the integrals in all variables are converging). It remains to deal with the first term in (38), we notice that $\theta$ is even and so

$$
\int \varphi\left(x^{\prime}\right) e^{-i \lambda x^{\prime} \zeta} \theta(\zeta) d x^{\prime} d \zeta=\lambda^{-1} \int \hat{\theta}\left(x^{\prime}\right) \varphi\left(x^{\prime} / \lambda\right) d x^{\prime}=\lambda^{-1} \pi-\int \hat{\theta}\left(x^{\prime}\right)\left(1-\varphi\left(x^{\prime} / \lambda\right)\right) d x^{\prime} .
$$

Since $\hat{\theta}$ is Schwartz, the last line clearly has an expansion of the form $\pi \lambda^{-1}+O\left(\lambda^{-\infty}\right)$ for some constant $C$, and combining with (39), we deduce that (37) is thus homogeneous of degree $\lambda^{-j-k-1}$ modulo $O\left(\lambda^{\infty}\right)$. This ends the proof of the fact that $K L$ is a classical pseudodifferential operator on $M$. 
Now, we compute the principal symbol. According to the discussion above, it is given by

$$
\begin{gathered}
-i(2 \pi)^{-2} \int \frac{1}{\zeta}\left(\sigma_{L}\left(y ; \xi^{\prime}, \mu\right)\left((1-\theta(\zeta)) \sigma_{K}\left(y ; \xi^{\prime}+\zeta, \mu\right)+\theta(\zeta) \zeta \sigma_{K}^{\prime}\left(y ; \xi^{\prime}, \zeta, \mu\right)\right) d \xi^{\prime} d \zeta\right. \\
+(2 \pi)^{-2} \pi \int \sigma_{L}\left(y, \xi^{\prime}, \mu\right) \sigma_{K}\left(y ; \xi^{\prime}, \mu\right) d \xi^{\prime}
\end{gathered}
$$

where $\zeta \sigma_{K}^{\prime}\left(y ; \xi^{\prime}, \zeta, \mu\right):=\sigma_{K}\left(y ; \xi^{\prime}+\zeta, \mu\right)-\sigma_{K}\left(y ; \xi^{\prime}, \mu\right)$. Now, it is straightforward to see that this is equal to (34) by using that the Fourier transform of the Heaviside function is the distribution $\pi \delta-i / \zeta$. Notice that the integral (34) makes sense since $\sigma_{K}, \sigma_{L}$ are $L^{2}$ in the $\xi^{\prime}$ variable.

\section{REFERENCES}

[1] M. F. Atiyah and I. M. Singer, The index of elliptic operators on compact manifolds., Bull. Amer. Math. Soc. 69 (1963) 422-433.

[2] E. Aubry, C. Guillarmou, Conformal harmonic forms, Branson-Gover operators and Dirichlet problem at infinity,, to appear Journ. Eur. Math. Soc., arXiv:0808.0552.

[3] R. Beals, P. Greiner, Calculus on Heisenberg manifolds, Annals of Mathematics studies no 119, Princeton University Press.

[4] N. Berline, E. Getzler, M. Vergne, Heat kernel and Dirac operators, 2004 edition of Vol 298 Grundlehren der mathematishen Wissenschaften 1992, Springer Verlag.

[5] B. Booss-Bavnbek, M. Lesch and C. Zhu, The Calderón projection: New definition and applications, Journal of Geometry and Physics 59 (2009), No. 7, 784-826 .

[6] B. Booss-Bavnbek and K. P. Wojciechowski, Elliptic boundary problems for Dirac operators, Birkhäuser Boston, Inc., Boston, MA 1993.

[7] B. Bojarski, The abstract linear conjugation problem and Fredholm pairs of subspaces, In Memoriam I. N. Vekua (Tbilisi Univ. 1979) 45-60.

[8] M. Braverman, New proof of the cobordism invariance of the index, Proc. Amer. Math. Soc. 130 (2002) 1095-1101.

[9] A. P. Calderón, Boundary value problems for elliptic equations, 1963 Outlines Joint Sympos. Partial Differential Equations (Novosibirsk, 1963) 303-304.

[10] S. Y. Cheng and S. T. Yau, On the existence of a complete Kähler metric on noncompact complex manifolds and the regularity of Fefferman's equation, Comm. Pure Appl. Math. 33 (1980), no. 4, 507-544.

[11] H. Donnelly and C. Fefferman, $L^{2}$-cohomology and index theorem for the Bergman metric, Ann. of Math. 118 (1983), no. 3, 593-618.

[12] C. L. Epstein, Subelliptic Spin $_{\mathbb{C}}$ Dirac operators. I, Ann. of Math. (2) 166 (2007), no. 1, 183-214.

[13] C. L. Epstein, Subelliptic Spin $_{\mathbb{C}}$ Dirac operators. II. Basic estimates, Ann. of Math. (2) 166 (2007), no. 3, $723-777$.

[14] C. L. Epstein and R. Melrose, Shrinking tubes and the d-bar Neumann problem, preprint available online at http://www.math.upenn.edu/ cle/papers/index.html.

[15] C. Fefferman The Bergman kernel and biholomorphic mappings of pseudoconvex domains. Invent. Math. 26 (1974), 1-65.

[16] C. Fefferman and C. R. Graham, Conformal invariants, SMF Astérisque, hors série (1985), 95-116.

[17] C. Fefferman and C. R. Graham, The ambient metric, preprint arXiv:0710.0919.

[18] P. Gilkey, The residue of the local eta function at the origin, Math. Ann. 240 (1979), no. 2, $183-189$.

[19] C. R. Graham and J. M. Lee, Einstein metrics with prescribed conformal infinity on the ball, Adv. Math. 87 (1991), no. 2, 186-225.

[20] C.R. Graham, M. Zworski, Scattering matrix in conformal geometry, Invent. Math. 152 (2003), 89-118.

[21] A. Grigis, J. Sjöstrand, Microlocal analysis for differential operators, Lecture note series 196 (1994) London Math. Soc., Cambridge Univ. Press.

[22] C. Guillarmou, Meromorphic properties of the resolvent for asymptotically hyperbolic manifolds, Duke Math. J. 129 no. 1 (2005), 1-37.

[23] C. Guillarmou and A. Hassell, Resolvent at low energy and Riesz transform for Schrödinger operators on asymptotically conic manifolds. I. Math. Ann. 341 (2008), no. 4, 859-896. 
[24] C. Guillarmou, S. Moroianu and J. Park, Eta invariant, Dirac operator and odd Selberg zeta function on convex co-compact hyperbolic manifolds, Adv. Math. 225 (2010), no 5, 2464-2516.

[25] N. Hitchin, Harmonic spinors. Advances in Math. 14 (1974), 1-55.

[26] L. Hörmander, The analysis of linear partial differential operators, I, Distribution theory and Fourier analysis. Springer-Verlag, Berlin, 2003

[27] M. Joshi, A. Sá Barreto, Inverse scattering on asymptotically hyperbolic manifolds, Acta Math. 184 (2000), $41-86$.

[28] P. Kirk and M. Lesch, The $\eta$-invariant, Maslov index, and spectral flow for Dirac-type operators on manifolds with boundary, Forum Math. 16 (2004), no. 4, 553-629.

[29] B.H. Lawson, M-L. Michelsohn, Spin geometry. Princeton Mathematical Series, 38. Princeton University Press, Princeton, NJ, 1989.

[30] M. Lesch, Deficiency indices for symmetric Dirac operators on manifolds with conic singularities, Topology 32 611-623.

[31] P. Loya, Geometric BVPs, Hardy spaces, and the Cauchy integral and transform on regions with corners, J. Differential Equations 239 (2007), no. 1, 132-195.

[32] P. Loya and J. Park, On the gluing problem for the spectral invariants of Dirac operators, Adv. Math. 202 (2006), no. 2, 401-450.

[33] R. Mazzeo, Elliptic theory of differential edge operators I, Comm. Partial Diff. Equations 16 (1991), no. 10, $1615-1664$.

[34] R. Mazzeo, R. Melrose, Meromorphic extension of the resolvent on complete spaces with asymptotically constant negative curvature, J. Funct. Anal. 75 (1987), 260-310.

[35] R. B. Melrose, Calculus of conormal distributions on manifolds with corners, Int. Math. Res. Not. 3 (1992), 51-61.

[36] R. B. Melrose, The Atiyah-Patodi-Singer index theorem (AK Peters, Wellesley, 1993).

[37] R. B. Melrose, Differential analysis on manifolds with corners, book in preparation, available online at www-math.mit.edu/ rbm/book.html.

[38] S. Moroianu, Cusp geometry and the cobordism invariance of the index, Adv. Math. 194 (2005), 504-519.

[39] W. Müller, A. Strohmaier Scattering of low energies on manifolds with cylindrical ends and stable systoles, GAFA, to appear.

[40] L. I. Nicolaescu, The Maslov index, the spectral flow, and decompositions of manifolds, Duke Math. J. 80 (1995), no. 2, 485-533.

[41] L. I. Nicolaescu, On the cobordism invariance of the index of Dirac operators, Proc. AMS 125 (1997), 2797-2801.

[42] S. G. Scott and K. P. Wojciechowski, The $\zeta$-determinant and Quillen determinant for a Dirac operator on a manifold with boundary, Geom. Funct. Anal. 10 (2000), no. 5, 1202-1236.

[43] R. T. Seeley, Singular integrals and boundary value problems, Amer. J. Math. 88 (1966), 781-809.

[44] R. T. Seeley, Topics in pseudo-differential operators, 1969 Pseudo-Diff. Operators (C.I.M.E., Stresa, 1968) $167-305$.

[45] M. Wodzicki, Local invariants of spectral asymmetry. Invent. Math. 75 (1984), no. 1, 143-177.

DMA, U.M.R. 8553 CNRS, Ecole Normale Supérieure, 45 rue D’Ulm, F 75230 Paris Cedex 05, FRANCE

E-mail address: cguillar@dma.ens.fr

Institutul de Matematic $\breve{A}$ al Academiei Române, P.O. Box 1-764, RO-014700 Bucharest, RoMANIA

E-mail address: moroianu@alum.mit.edu

School of Mathematics, Korea Institute for Advanced Study, 207-43, Hoegiro 87, DongDaemun-Gu, Seoul 130-722, Republic of Korea

E-mail address: jinsung@kias.re.kr 